\title{
The Thurston polytope for four-stranded pretzel links
}

\author{
JOAN E LICATA
}

\begin{abstract}
In this paper we use Heegaard Floer link homology to determine the dual Thurston polytope for pretzel links of the form $P\left(-2 r_{1}-1,2 q_{1},-2 q_{2}, 2 r_{2}+1\right), r_{i}, q_{i} \in \mathbb{Z}^{+}$. We apply this result to determine the Thurston norms of spanning surfaces for the individual link components, and we explicitly construct norm-realizing surfaces for the homology classes which are vertices of the Thurston polytope.
\end{abstract}

57M27; 53D99, 57R58, 57M25

\section{Introduction}

The Thurston norm is a fundamental measure of the complexity of second homology classes in a three-manifold. When the manifold is a knot complement, the second homology is spanned by any Seifert surface for the knot $K$, and the genus of $K$ is defined to be the least genus of any Seifert surface for $K$. In the case of a link in the three-sphere, the analogous objects are surfaces whose boundaries lie on the components of the link. In this paper we study the two-component pretzel links $P\left(-2 r_{1}-1,2 q_{1},-2 q_{2}, 2 r_{2}+1\right), r_{i}, q_{i} \in \mathbb{Z}^{+}$, which we denote by $P_{q_{1}, r_{1}, q_{2}, r_{2}}$, and we determine the dual Thurston polytopes of their complements.

Let $B_{T}^{*}\left(P_{q_{1}, r_{1}, q_{2}, r_{2}}\right)$ be the unit ball with respect to the dual Thurston norm on $H_{1}\left(S^{3}-\right.$ $\left.P_{q_{1}, r_{1}, q_{2}, r_{2}} ; \mathbb{R}\right)$. We specify as a basis for $H_{1}\left(S^{3}-P_{q_{1}, r_{1}, q_{2}, r_{2}} ; \mathbb{R}\right)$ the set of oriented meridians of the link components. Identify the meridians with the coordinate vectors of $\mathbb{R}^{2}$ so that the meridian of the unknotted component $U$ is the vector $(1,0)$, and the meridian of the knotted component $K$ is the vector $(0,1)$. Let $r_{B}=\max \left(r_{1}, r_{2}\right)$, $r_{S}=\min \left(r_{1}, r_{2}\right), q_{B}=\max \left(q_{1}, q_{2}\right)$, and $q_{S}=\min \left(q_{1}, q_{2}\right)$. In the special case $q_{1}=q_{2}=q$ and $r_{1}=r_{2}=r$, we denote $P_{q_{1}, r_{1}, q_{2}, r_{2}}$ by $P_{q, r}$.

Theorem $1 B_{T}^{*}\left(P_{q, r}\right)$ is the convex hull of the following points:

$$
\begin{array}{ll}
(-1,4 r-1) & (1,-4 r+1) \\
(2 q-3,2 q-3) & (-2 q+3,-2 q+3) \\
(2 q-1,2 q-3) & (-2 q+1,-2 q+3) \\
(2 q-1,-4 r+2 q-1) & (-2 q+1,4 r-2 q+1)
\end{array}
$$




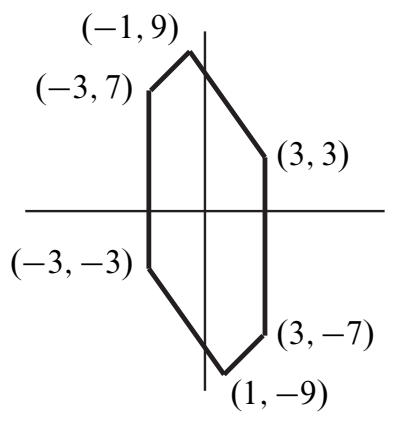

$(6,10)$

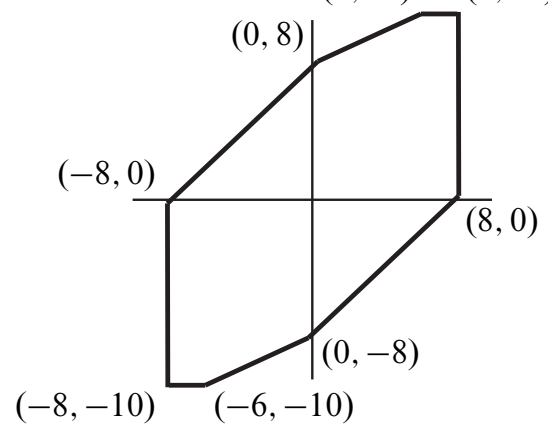

Figure 1: Left: $B_{T}^{*}\left(P_{2,2,2,3}\right)$. Right: $B_{T}^{*}\left(P_{4,1,5,3}\right)$.

$B_{T}^{*}\left(P_{q_{1}, r_{1}, q_{2}, r_{2}}\right)$ is the convex hull of the following points:

$$
\begin{array}{ll}
\left(q_{B}-q_{S}-1,2 r_{1}+2 r_{2}+q_{B}-q_{S}-1\right) & \left(-q_{B}+q_{S}+1,-2 r_{1}-2 r_{2}-q_{B}+q_{S}+1\right) \\
\left(q_{1}+q_{2}-3,2 r_{B}-2 r_{S}+q_{1}+q_{2}-3\right) & \left(-q_{1}-q_{2}+3,-2 r_{B}+2 r_{S}-q_{1}-q_{2}+3\right) \\
\left(q_{1}+q_{2}-1,2 r_{B}-2 r_{S}+q_{1}+q_{2}-3\right) & \left(-q_{1}-q_{2}+1,-2 r_{B}+2 r_{S}-q_{1}-q_{2}+3\right) \\
\left(q_{1}+q_{2}-1,-2 r_{1}-2 r_{2}+q_{1}+q_{2}-1\right) & \left(-q_{1}-q_{2}+1,2 r_{1}+2 r_{2}-q_{1}-q_{2}+1\right)
\end{array}
$$

(If $2 q$ (respectively, $q_{1}+q_{2}$ ) $\leq 2$, the points in the second line should be omitted.)

Figure 1 shows two examples of dual Thurston polytopes for links in this family.

McMullen [3] showed that the multivariable Alexander polynomial of a link may provide information about the dual Thurston polytope of the link complement. This result offers no information about links with trivial Alexander polynomial, but work of Ozsváth-Szabó [6] and Ni [4] has shown that link Floer homology detects the Thurston norm. We prove Theorem 1 by determining the filtration support for the Heegaard Floer link homology of $P_{q_{1}, r_{1}, q_{2}, r_{2}}$. The choice of this family of links is motivated by a famous example of a knot with trivial Alexander polynomial, the Kinoshita-Terasaka knot. The link $P_{1,1}$ differs from the Kinoshita-Terasaka knot by the resolution of a single crossing, and in fact the Alexander polynomial vanishes for all the links in the subfamily $P_{q, r}$ (Corollary 2$)$.

$B_{T}^{*}\left(P_{q_{1}, r_{1}, q_{2}, r_{2}}\right)$ is the dual norm ball in $\mathbb{R}^{2} \cong H_{1}\left(S^{3}-P_{q_{1}, r_{1}, q_{2}, r_{2}} ; \mathbb{R}\right)$ of the unit ball with respect to the Thurston norm on $H_{2}\left(S^{3}, P_{q_{1}, r_{1}, q_{2}, r_{2}} ; \mathbb{R}\right)$. Thus, the Thurston norm of a homology class in $H_{2}\left(S^{3}, P_{q_{1}, r_{1}, q_{2}, r_{2}} ; \mathbb{R}\right)$ represented by a unit vector $\mathbf{u}$ is half the length of the projection of $B_{T}^{*}\left(P_{q_{1}, r_{1}, q_{2}, r_{2}}\right)$ onto a line parallel to $\mathbf{u}$. Theorem 1 therefore implies the following: 
Corollary 1 The unknotted component of $P_{q_{1}, r_{1}, q_{2}, r_{2}}$ bounds a surface $F_{U}$ with Euler characteristic $1-q_{1}-q_{2}$, The knotted component of $P_{q_{1}, r_{1}, q_{2}, r_{2}}$ bounds a surface $F_{K}$ with Euler characteristic $-\max \left\{2 r_{1}+2 r_{2}+q_{B}-q_{S}-1,2 r_{B}-2 r_{S}+q_{1}+q_{2}-3\right\}$. $F_{U}$ and $F_{K}$ have maximal Euler characteristic in their respective homology classes.

When $2 r_{S}+1>q_{S}$, the complexity of $F_{K}$ equals the Thurston norm of the generator of $H_{2}\left(S^{3}, K\right)$. This equality (proved in Section 6.3) implies a minimal-complexity surface $\left(F_{K}, \partial F_{K}\right) \subset\left(S^{3}-P_{q, r}, \partial N(K)\right)$ which is also a minimal-complexity Seifert surface for $K$ in $S^{3}$. In particular, the links $P_{q, r}$ are split into two classes by the inequality $2 r+1 \gtrless q$, and within each class the Thurston norm of $F_{K}$ is controlled exclusively by one of $q$ or $r$ : provided that $2 r+1$ is great enough, the Euler characteristic of $F_{K}$ does not depend on the linking with the unknotted component. The opposite is true when $2 r+1<q$; then, the Euler characteristic of $F_{K}$ is a function solely of $q$.

We begin with a discussion of the Thurston norm (Section 2) and pretzel links (Section 3). In Section 4 we introduce a technique for building surfaces in link complements. We employ this to explicitly construct norm-realizing surfaces for the vertex classes of the Thurston polytope of $P_{q, r}$. Section 5 introduces the definitions and basic properties of Heegaard Floer link homology, and we prove Theorem 1 in the final section.

\subsection{Acknowledgements}

The author would like to thank Peter Ozsváth for his encouragement and support, and the following individuals have also been the source of many helpful conversations: John Baldwin, Elisenda Grigsby, Paul Melvin, Jiajun Wang, and Shaffiq Welji. Finally, the referee's feedback has been invaluable and is much appreciated.

\section{The Thurston norm}

In [10], Thurston defined a seminorm on the second homology of a three-manifold with boundary. This norm measures the minimal combinatorial complexity of a surface representing a fixed second homology class. When considering link complements, we will take the "Thurston norm of $L$ " to mean the Thurston norm of the manifold $S^{3}-L$

Definition 1 Let $S$ be an embedded surface with components $s_{i}$. The complexity $\chi_{-}(S)$ of a surface with components $s_{i}$ is given by

$$
\chi_{-}(S)=\sum_{i: \chi\left(s_{i}\right) \leq 0}-\chi\left(s_{i}\right)
$$

Algebraic 83 Geometric Topology, Volume 8 (2008) 
where $\chi\left(s_{i}\right)$ denotes the Euler characteristic of $s_{i}$.

Definition 2 For $\sigma \in H_{2}\left(S^{3}, L ; \mathbb{Z}\right)$, the Thurston norm of $\sigma$ is given by

$$
\|\sigma\|_{T}=\min \left\{\chi_{-}(S):[S]=\sigma\right\} .
$$

Thurston proved in [10] that the function $\|\cdot\|_{T}$ extends to a seminorm on $H_{2}\left(S^{3}, L ; \mathbb{R}\right)$ taking values in $\mathbb{R}$. In general, $\|\cdot\|_{T}$ is a true norm on an appropriate quotient of $H_{2}\left(S^{3}, L ; \mathbb{R}\right)$. ( $\|\cdot\|_{T}$ is also a true norm on the homology of all the examples considered in this paper.) In [1], Gabai showed that allowing minimal complexity immersed surfaces representing a fixed class preserves the Thurston norm of the class.

Fixing a basis for $\mathrm{H}_{2}\left(S^{3}, L ; \mathbb{R}\right)$ allows us to study the geometry of the Thurston norm. Specifically, choose $|L|$ surfaces, each of which spans one component of the link and is disjoint from the others. The surfaces are oriented so that the induced boundary orientations agree with the orientations on the link components, and the corresponding homology classes form a basis for $\mathrm{H}_{2}\left(S^{3}, L\right)$. Identify each basis element with a unit vector on a coordinate axis of $\mathbb{R}^{|L|}$.

Definition 3 The Thurston polytope is the unit ball with respect to the Thurston norm under this identification of $\mathbb{R}^{|L|}$ with $H_{2}\left(S^{3}, L ; \mathbb{R}\right)$.

The Thurston norm ball is a symmetric polytope [10], and we denote it by $B_{T}(L)$. As a norm on a finite-dimensional vector space, the Thurston norm has a natural dual.

Definition 4 The dual Thurston norm $\|\tau\|_{T}^{*}$ for $\tau \in H^{2}\left(S^{3}, L ; \mathbb{R}\right) \cong H_{1}\left(S^{3}-L ; \mathbb{R}\right)$ is given by

$$
\|\tau\|_{T}^{*}=\sup \{|\tau(\sigma)|\}
$$

where the supremum is taken over $\sigma$ with $\|\sigma\|_{T}=1$.

The unit ball with respect to $\|\cdot\|_{T}^{*}$ is the dual polytope of the Thurston norm ball; we denote the dual Thurston norm ball by $B_{T}^{*}(L)$.

The Alexander polynomial $\Delta(L)$ of a link $L$ has one variable $x_{i}$ associated to the meridian $\mu_{i}$ of each component of $L$. Sending $\left(\left\{x_{i}\right\}, \cdot\right)$ to $\left(\left\{\mu_{i}\right\},+\right)$ identifies each monomial summand of $\Delta(L)$ with an element of $H_{1}\left(S^{3}-L\right)$. The convex hull of these lattice points is known as the Newton polytope of $L$. A result of McMullen [3] relates $B_{T}^{*}(L)$ to the Newton polytope, but in general $\Delta(L)$ does not determine $B_{T}^{*}(L)$. Heegaard Floer link homology categorifies the multivariable Alexander polyonomial, and this stronger invariant determines the dual Thurston norm ball completely [6]. This relationship will be described precisely in Section 5.3. 


\section{Pretzel links}

Let $B_{a_{i}}$ be the two-strand braid $\sigma^{a_{i}}$, where $\sigma$ is the braid group generator and $a_{i} \in \mathbb{Z}$. Adding bridges to cyclically connect the elements of a collection $\left\{B_{a_{i}}\right\}_{i=1}^{n}$ gives the pretzel link $P\left(a_{1}, a_{2} \ldots a_{n}\right)$. When the pretzel link is in $n$-bridge position with the connecting arcs forming the bridges, we say the projection is standard. Figure 2 shows an example of the pretzel link $P(-5,4,-4,5)$ in the standard projection.

If the $a_{i}$ all have the same sign, then the link is alternating; in this case, the Alexander polynomial of the link determines its Thurston norm (see Ozsváth and Szabó [6]). For the remainder, we restrict our attention to 4-tuples of twist coefficients whose signs alternate and parities do not. Although the four coefficients determine the link, there are equivalence classes of 4-tuples that give isotopic links. In particular, $P\left(2 r_{1}+1,-2 q_{1}, 2 q_{2},-2 r_{2}-1\right)$ and $P\left(-2 q_{1}, 2 r_{1}+1,-2 r_{2}-1,2 q_{2}\right)$ are isotopic, although typically $P\left(2 r_{1}+1,-2 q_{1}, 2 q_{2},-2 r_{2}-1\right)$ and $P\left(-2 q_{1}, 2 r_{1}+1,2 q_{2},-2 r_{2}-1\right)$ are not.

The Thurston norm of $L$ depends only on the homeomorphism type of the manifold and is therefore independent of the orientation of the link. Since $B_{T}^{*}(L)$ is symmetric with respect to the origin, it is independent of the choice of orientation for the link components. Furthermore, the complements of a link and its mirror (the link $\bar{L}$ formed by changing every crossing in a planar projection of $L$ ) are homeomorphic. Since the Thurston norm of a link and its mirror agree, it suffices to consider only $P\left(-2 r_{1}-1,2 q_{1},-2 q_{2}, 2 r_{2}+1\right), q_{i}, r_{i} \in \mathbb{Z}^{+}$, which we denote by $P_{q_{1}, r_{1}, q_{2}, r_{2}}$. This four-parameter family of links always has one knotted component, which we denote by $K$, and one unknotted component, which we denote by $U$. For simplicity, we write $P_{q, r}$ for $P_{q, r, q, r}$.

\section{Surfaces in the link complement}

Classes in the basis for $H_{2}\left(S^{3}, P_{q_{1}, r_{1}, q_{2}, r_{2}}\right)$ chosen above are Poincare dual to the meridians of the various link components. We refer to surfaces representing these classes as spanning surfaces for the link components. Spanning surfaces are a generalization of Seifert surfaces, as the former may be punctured by other components of the link.

Rephrasing Corollary 1 in the language of Section 2, we have the following:

Corollary 1 There exist surfaces $F_{U}$ and $F_{K}$ in $S^{3}-P_{q_{1}, r_{1}, q_{2}, r_{2}}$ satisfying the following:

$$
\begin{aligned}
& -\chi\left(F_{U}\right)=\|(1,0)\|_{T}=q_{1}+q_{2}-1 \\
& -\chi\left(F_{K}\right)=\|(0,1)\|_{T}=\max \left\{2 r_{1}+2 r_{2}+q_{B}-q_{S}-1,2 r_{B}-2 r_{S}+q_{1}+q_{2}-3\right\} .
\end{aligned}
$$




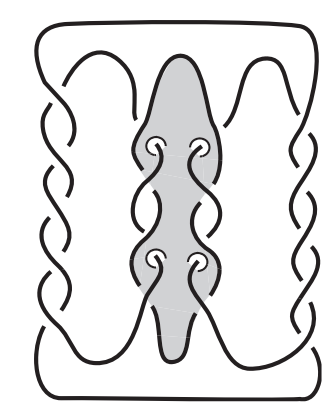

Figure 2: $P_{2,2}$ is shown in standard position with a minimal-complexity spanning surface for the unknotted component $U$. The knotted component $K$ punctures $F_{U}$ four times.

Any spanning surface for the unknotted component $U$ represents the homology class $(1,0)$, and according to Corollary $1,\|(1,0)\|_{T}=q_{1}+q_{2}-1$. A surface with this complexity is easily realized: in the standard projection of $P_{q_{1}, r_{1}, q_{2}, r_{2}}, U$ bounds a disc in the plane which is punctured precisely $q_{1}+q_{2}$ times by $K$. Figure 2 illustrates $F_{U}$ for $P_{2,2}$.

\subsection{Morse movies and $\boldsymbol{F}_{K}$}

Turning to $F_{K}$, we first consider minimal-complexity representatives of $(0,1) \in$ $H_{2}\left(S^{3}, P_{q, r}\right)$. As indicated in Corollary $1,\|(0,1)\|_{T}=\max \{4 r-1,2 q-3\}$. The two cases correspond to variations in the shape of $B_{T}^{*}\left(P_{q, r}\right)$; if $4 r-1<2 q-3$, the dual Thurston polytope is eight-sided, but if $4 r-1 \geq 2 q-3$, then $B_{T}^{*}\left(P_{q, r}\right)$ is the convex hull of a proper subset of the points listed in Theorem 1 . Although $F_{U}$ may be realized as a punctured disc in the plane of the paper, $F_{K}$ is best presented as a movie.

Given a standard projection of $P_{q, r}$, there is a natural Morse function $f: F_{K} \rightarrow[0,1]$ given by height on the page. A Morse movie is a sequence of frames, each of which is the preimage $f^{-1}(x)$ of some generic $x \in[0,1]$. Any frame differs from the previous one by isotopy or by a handle addition corresponding to a critical point of the Morse function. Thus, the movie shows a descending sequence of horizontal slices through $F_{K}$ that captures the topology of the surface. In our Morse movie of $F_{K}$, the initial frames will differ only by isotopies dictated by the twisting of the strands. Subsequently we will perform one-handle additions in order to arrive at a frame consisting of simple closed curves that may be capped off by two-handles.

Viewing the vertical strands of the link as a braid $B$ in $\mathbb{C} \times[0,1]$ defines an isotopy $\mathfrak{B}$ of $\mathbb{C}$. The braid group generator $\sigma_{i}$ maps to an isotopy $\mathfrak{b}_{i}$ of the marked plane which 


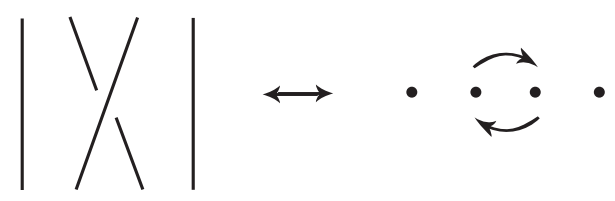

Figure 3: The braid generator $\sigma_{i}$ corresponds to $\mathfrak{b}_{i}$, an isotopy of the plane that interchanges two points by rotating a small neighborhood of the pair.

interchanges the $i^{\text {th }}$ and $(i+1)^{\text {th }}$ points by clockwise rotation of a neighborhood of the pair. $\mathfrak{b}_{i}$ is the identity away from a neighborhood of these two points, and multiplication in the braid group corresponds to composition of the associated $\mathfrak{b}_{i}$. See Figure 3 for an example. The isotopy $\mathfrak{B}$ thus defined has the following property. Consider $B$ as a collection of embedded arcs in $\mathbb{C} \times[0,1]$ and let $\mathbf{p}=B \cap\{1\}$. For all $s \in[0,1]$, $\mathfrak{B}: \mathbb{C} \times[0,1] \rightarrow \mathbb{C}$ satisfies $\mathfrak{B}(\mathbf{p}, s)=B \cap(\mathbb{C} \times(1-s))$. Intuitively, if the isotopy parameter is identified with one minus the $z$ coordinate, $\mathfrak{B}$ makes $2 n$ colinear points in the plane trace out $B$ in $\mathbb{C} \times[0,1]$. This map is well-defined up to isotopy fixing $\mathbf{p} \times\{0,1\}$.

Returning to the construction of $F_{K}$, let the first frame in the Morse movie be $f^{-1}(1-\epsilon)$. This slice taken immediately below the bridges of the standard projection consists of three disjoint curves parallel to the bridges as well as two points where the slice intersects $U$. (See Figure 5.) $\mathfrak{B}$ is defined via the marked points, but it acts on the entire plane. The image of the initial frame under $\mathfrak{B}$ may be thought of as a horizontal slice taken immediately below the braid $B$ in the standard projection. This frame is clearly isotopic to the original one, but we will perform a sequence of saddle moves on successive frames which correspond to one-handle additions in the surface and change its Euler characteristic. The curves in each frame inherit co-orientations from the surface, and these are indicated by arrows transverse to the curves in the diagram; the saddle resolutions must be consistent with these orientations in order to yield an orientable surface.

The frames created by this braid action will share some general features independent of the values of $q_{i}$ and $r_{i}$, and we introduce three types of saddle moves which may be applied to these frames. See Figure 4 for an illustration of these moves.

(1) Winding $K$ around $U$ (the braid action $\sigma_{3}^{2 q} \sigma_{5}^{-2 q}$ ) produces a pair of spirals in the center of the frame; define $S_{1}$ to be the saddle move applied at a pair of nearest points on these spirals.

(2) The twisting of $K$ with itself creates spirals on the left and right sides of the frame; define $S_{2}$ to be the saddle move that joins an arc from a lateral spiral to a nearest arc from the central spiral.

Algebraic $8 \mathcal{G}$ Geometric Topology, Volume 8 (2008) 


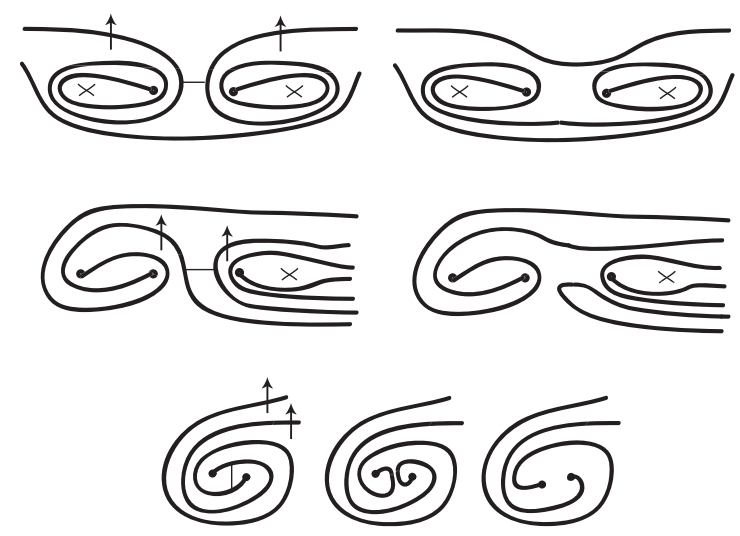

Figure 4: Top: $S_{1}$. Center: $S_{2}$ on the left side of the frame. Bottom: $S_{3}$ on left side of the frame. Each strand of $U$ is represented by an $\mathrm{x}$ and each strand of $K$ by a dot. The arrows indicate the co-orientations of the curves, and the line segments indicate where the saddle is performed.

(3) Let $S_{3}$ be the saddle move in the center of either of the lateral spirals which unwinds it by a half-twist.

When $4 r-1=2 q-3$, performing $q S_{1}$ moves and 2(q-1) $S_{2}$ moves yields a frame with $q-1$ simple closed curves and three arcs. However, the arcs can be connected to form an additional two simple closed curves by extending the surface to the lower bridges of the link. Capping off all the simple closed curves with two-handles gives a surface bounding $K$ with Euler characteristic $-2 q+3$. Figure 5 shows an example of a movie presentation for $F_{K}$.

If $4 r-1>2 q-3$, however, the concentric circles will be exhausted before the lateral spirals, and applying $S_{3}$ to the lateral spirals yields a surface of Euler characterisitic $-2 q+3-2(2 r-q+1)=-4 r+1$, as desired.

Finally, when $4 r-1<2 q-3$ we restrict the action of the braid group on the first frame to $\sigma_{1}^{-2 r-1} \sigma_{3}^{4 r+2} \sigma_{5}^{-4 r-2} \sigma_{7}^{2 r+1}$. Each additional full twist of $U$ around $K$ $\left(\sigma_{3}^{2}\right.$ or $\sigma_{5}^{-2}$ ) introduces a puncture in the surface rather than a further isotopy of the leading edge. Each puncture lowers the Euler characteristic by one, so applying Morse moves as in the $4 r-1=2 q-3$ case then yields a Seifert surface for $K$ whose Euler characteristic is $1-4 r-(2 q-4 r-2)=-2 q+3$.

Although we described a construction for $P_{q, r}$, it extends to $P_{q_{1}, r_{1}, q_{2}, r_{2}}$ with the stipulation that equal powers of $\sigma_{3}$ and $\sigma_{5}^{-1}$ act on the first frame; any additional twists of $U$ around $K$ puncture the surface. This power is $2 q_{S}$ if $2 r_{S} \geq q_{S}-1$, and 


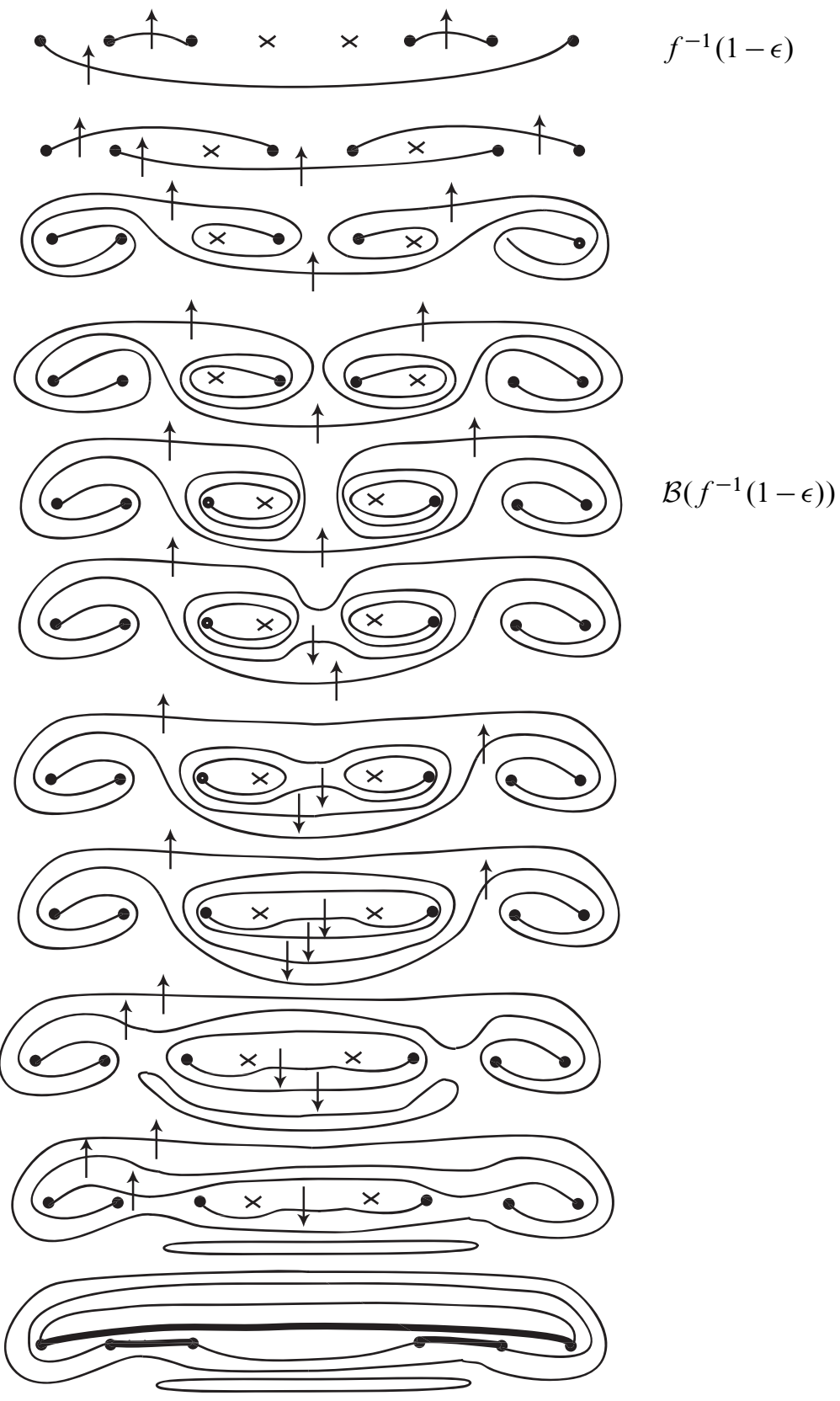

Figure 5: The figure is a movie presentation of $F_{K}$ for $P_{3,1}$. The x's are strands of $U$, and the dots are strands of $K$. The surface has seven saddles and requires four death moves, so the Euler characteristic is -3 . The arrows indicate the co-orientations of the curves.

Algebraic ${ }^{3} \mathcal{G}$ Geometric Topology, Volume 8 (2008) 

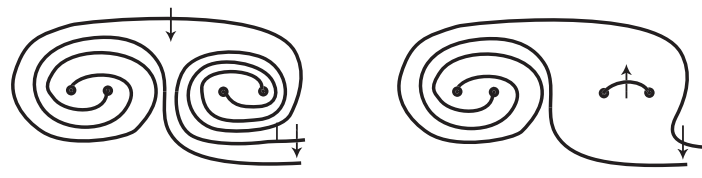

Figure 6: A spiral between curves bounded by $U$ and $K$ with arms of opposite co-orientation. The arc in the left figure indicates an $S_{4}$ saddle resolution, and the right-hand picture shows the result of performing this resolution and an isotopy to simplify the diagram.

the power is $4 r_{S}+2$ if $2 r_{S}<q_{S}-1$. Maximal application of $S_{1}$ and $S_{2}$ moves, followed by $S_{3}$ moves if necessary, then yields an orientable surface with the desired complexity.

\subsection{Vertex classes for $B_{T}\left(P_{q, r}\right)$}

The surfaces constructed in the previous section are minimal-complexity representatives for the chosen basis of $\mathrm{H}_{2}\left(S^{3}, P_{q, r}\right)$, but constructing Thurston norm-realizing surfaces for arbitrary second homology classes requires minimal-complexity representatives of all the vertices of the Thurston polytope. By definition, representatives of the vertex classes generate all minimal surfaces under linear combination over $\mathbb{R}$. These linear combinations are formal objects unless one restricts to $\mathbb{Z}$ coefficients, in which case they are surfaces which maximize Euler characteristic. Up to sign, there are four classes to consider, and we construct minimal representatives for the remaining two vertex classes of $B_{T}\left(P_{q, r}\right)$.

The remaining faces in $B_{T}^{*}\left(P_{q, r}\right)$ have slopes 1 and $(q-2 r-1) / q$ if $2 r>q-1$ and 1 and $(q-2 r-1) /(q-1)$ if $2 r<q-1$. (If $2 r=q-1, B_{T}^{*}\left(P_{q, r}\right)$ has only six faces.) We first consider the class $(-1,1)$ and begin as above, letting the braid act on curves parallel to the upper bridges. Since both $U$ and $K$ bound, the spiral in the center of the diagram has twice as many arms as in the movie for $F_{K}$. We introduce a fourth resolution, $S_{4}$, which will be useful in the construction.

When the curves bounded by $U$ and $K$ have the same co-orientation, the two arms of the central spirals will have opposite co-orientations as depicted in Figure 6. Resolving along the arc indicated in the figure allows the entire spiral to vanish under isotopy.

Performing an $S_{4}$ saddle move on each of the central spirals and $2 r S_{3}$ resolutions on each of the lateral spirals yields a frame where the lower bridges may be glued to the endpoints of the curves. This produces two simple closed curves, and after capping these, the surface has the maximum possble Euler characteristic of $4 r$. See Figure 7 for an example of the construction. 

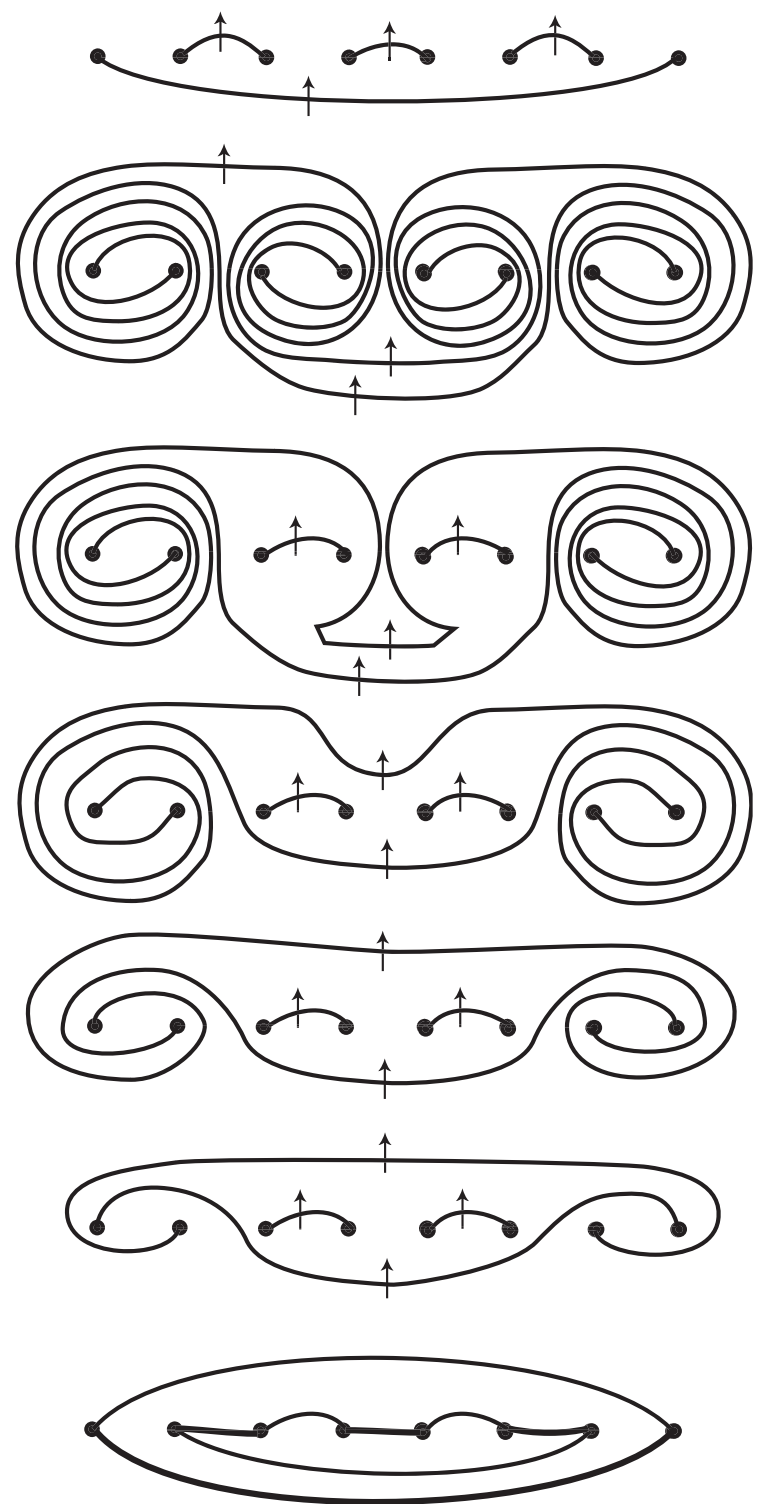

Figure 7: The $(-1,1)$ surface for $P_{2,2}$. The first two frames show $f^{-1}(1-\epsilon)$ and $\mathfrak{B}\left(f^{-1}(1-\epsilon)\right)$, and the successive frames indicate the result of the saddle resolutions. 
Next, we consider the final vertex class for the $2 r>q-1$ case, which is $(2 r-q+1, q) \in$ $\mathrm{H}_{2}\left(S^{3}, P_{q, r}\right)$. In the initial diagram, each point bounds a collection of parallel curves instead of a single one, and we represent this by labelling a single curve with the appropriate multiplicity before letting the braid act to yield the second frame shown. Performing $2 q r+2 q-2 r-1 S_{1}$ resolutions removes all the vertical curves from the center of the diagram, and performing $2 q r S_{2}$ resolutions on each side removes all the vertical curves between the central and lateral spirals. The frame can then be glued to the lower bridges, creating $2 q r+2 q$ simple closed curves so that the resulting surface has Euler characteristic $2 r+1-4 q r$, as desired. See Figure 8 for a diagram indicating this construction.

When $2 r<q-1$, the final vertex class is $(2 r-q+1, q-1)$. As in the $(-1,1)$ case, we begin by simplifying the central spirals using $S_{4}$ resolutions. Because the $K$ and $U$ curves have differrent multiplicities, the $U$ curves available for an $S_{4}$ resolution will be exhausted before the $K$ curves will. The coefficient of the $U$ multicurve, $q-2 r-1$, bounds the number of $S_{4}$ resolutions on each side. (Note that this is the absolute value of the $U$ coefficient applied to the curve with with opposite co-orientation.) Apply $2 q r S_{1}$ resolutions to the remaining central spirals, and $2 r q-2 r S_{2}$ resolutions to each side. Capping off the $2 q r-2 r+2 q-2$ simple closed curves yields a surface with Euler characteristic $6 r-4 q r$, which is maximal. An example is shown in Figure 9.

\section{Heegaard Floer homology}

In order to prove Theorem 1, we will apply a result of Oszváth and Szabó relating $B_{T}^{*}(L)$ to the Heegaard Floer homology of the link $L$. We supply here a minimum of background on Heegaard Floer theory, and we refer the reader to work of Ozsváth and Szabó $[8 ; 7 ; 5 ; 6]$ for details.

\subsection{The Heegaard diagram and $\pi_{2}(x, y)$}

Suppose that $L$ is a link in $S^{3}$. Let $\Sigma$ be a genus- $g$ surface decorated with simple closed curves $\left\{\alpha_{i}\right\}_{i=1}^{g+|L|-1}$ and $\left\{\beta_{i}\right\}_{i=1}^{g+|L|-1}$ and $|L|$-tuples of basepoints $\mathbf{z}=\left\{z_{i}\right\}_{i=1}^{|L|}$ and $\mathbf{w}=\left\{w_{i}\right\}_{i=1}^{|L|}$ satisfying the following:

- the $\alpha$ (respectively, $\beta$ ) curves are mutually disjoint;

- the $\alpha$ and $\beta$ curves intersect transversely;

- the $\left\{\alpha_{i}\right\}_{i=1}^{g+|L|-1}$ and $\left\{\beta_{i}\right\}_{i=1}^{g+|L|-1}$ each generate a rank- $g$ sublattice of $H_{1}(\Sigma)$; 

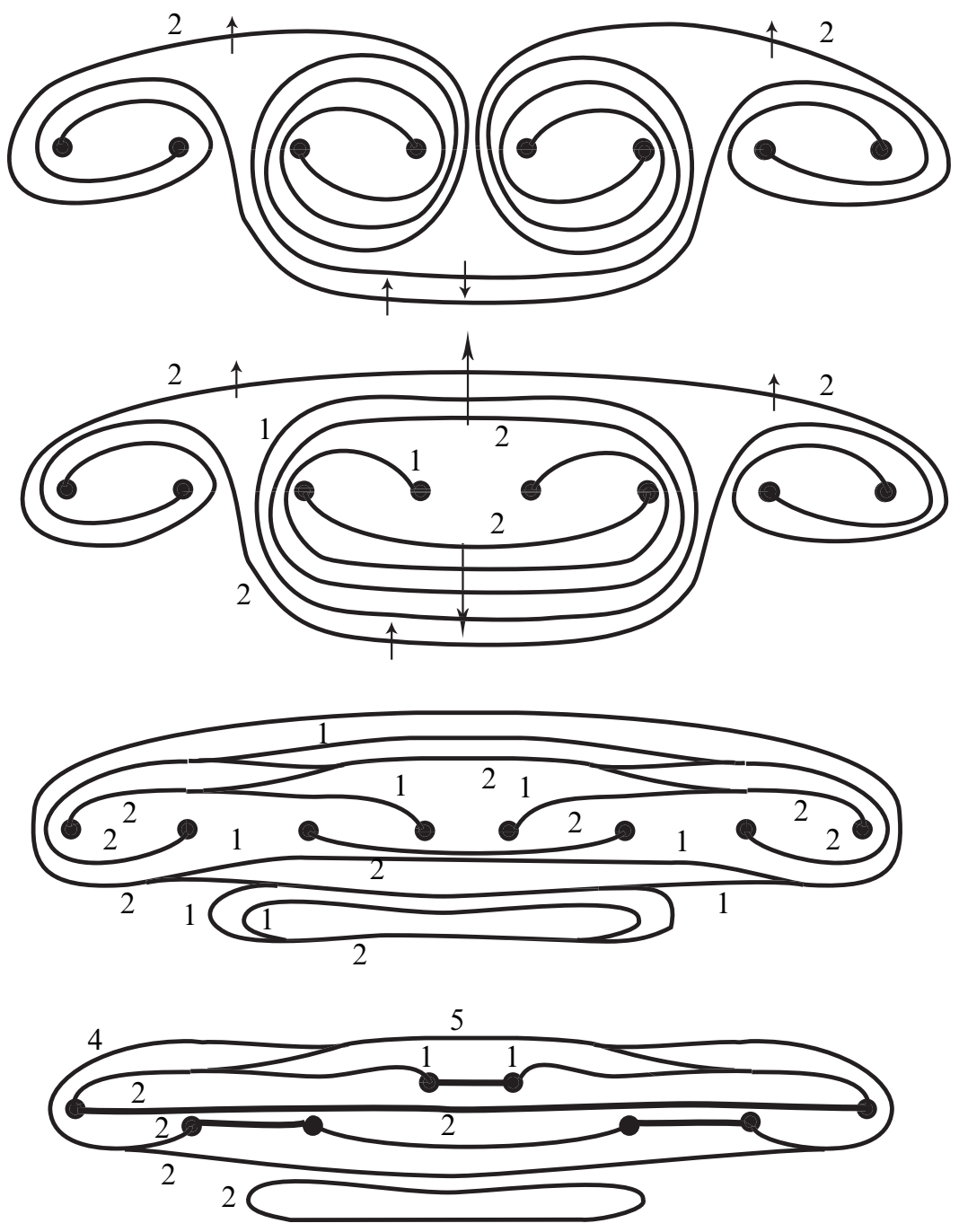

Figure 8: A schematic diagram for the minimal surface representing $(1,2) \in$ $H_{2}\left(P_{2,1}\right)$ Curves are labeled with their muliplicty, with a train-track convention at splices.

- the Heegaard diagram induced by any pair of linearly independent subsets of the attaching curves specifies $S^{3}$;

- each component of $\Sigma-\left\{\alpha_{i}\right\}$ (respectively, $\left.\Sigma-\left\{\beta_{i}\right\}\right)$ contains a pair $\left(z_{i}, w_{i}\right)$. 

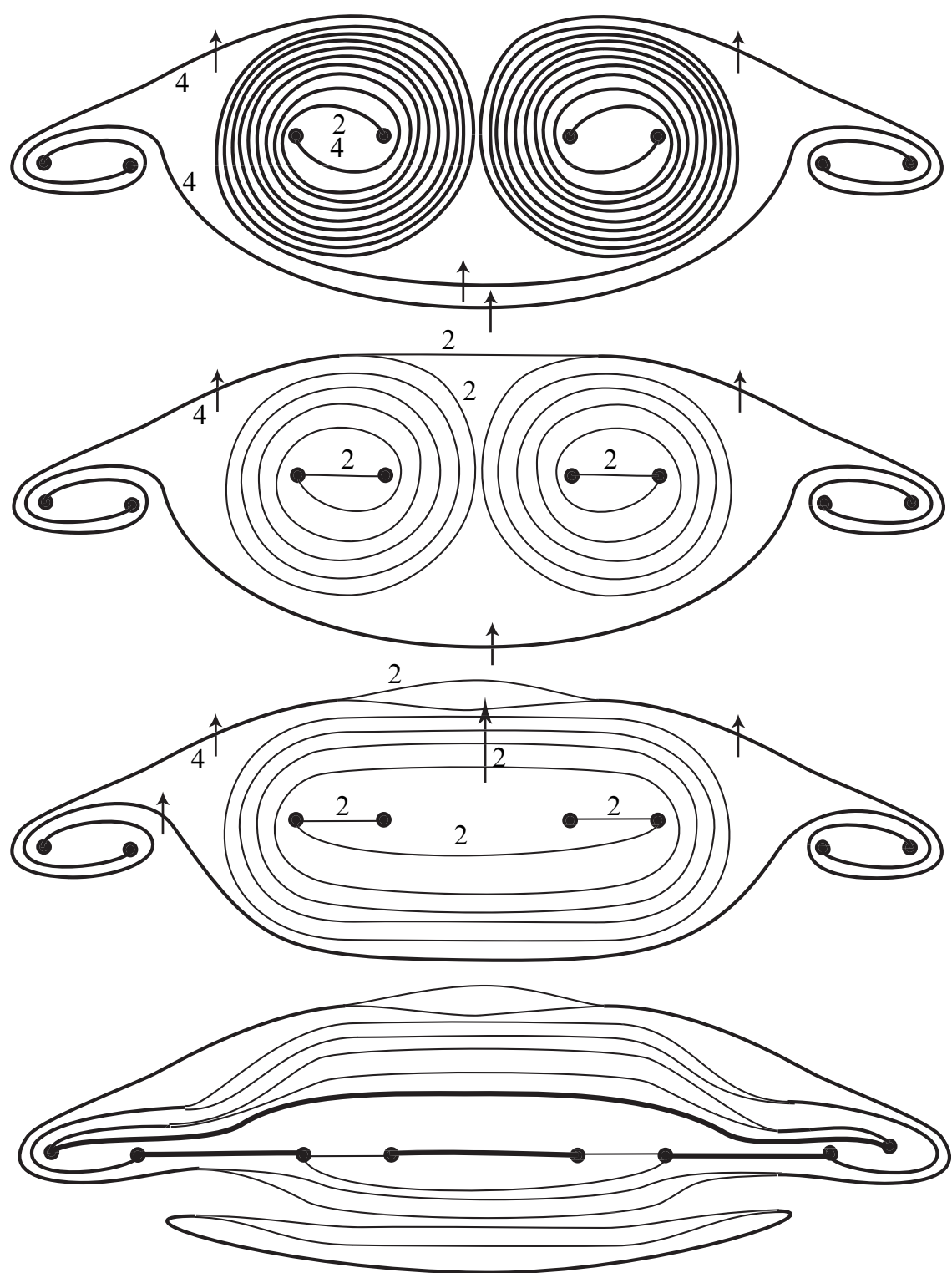

Figure 9: A schematic diagram for the minimal representative of $(-2,4) \in H_{2}\left(S^{3}, P_{5,1}\right)$.

Each basepoint pair specifies a link component in the following manner. One may connect each $\left(z_{i}, w_{i}\right)$ pair by two arcs, one in the complement of the $\left\{\alpha_{i}\right\}$ and one in 
the complement of the $\left\{\beta_{i}\right\}$. Pushing these arcs into the corresponding handlebodies gives a simple closed curve $L_{i}$ in $S^{3}$.

Definition 5 The pointed Heegaard diagram $\left(\Sigma,\left\{\alpha_{i}\right\},\left\{\beta_{i}\right\}, \mathbf{z}, \mathbf{w}\right)$ is compatible with $L$ if $L$ is isotopic in $S^{3}$ to the link formed by the simple closed curves specified by all the $\left(z_{i}, w_{i}\right)$.

The $n$-fold symmetric product of a space $X, \operatorname{Sym}^{n}(X)$, is the quotient space of the product $X^{n}$ under the action of the symmetric group $\mathfrak{S}_{n}$. Let $\mathbb{T}_{\alpha}$ (respectively, $\mathbb{T}_{\beta}$ ) be the image in $\operatorname{Sym}^{g+|L|-1}(\Sigma)$ of the product $\alpha_{1} \times \alpha_{2} \ldots \times \alpha_{g+|L|-1}\left(\beta_{1} \times \beta_{2} \ldots . \times\right.$ $\left.\beta_{g+|L|-1}\right)$. Since the $\alpha$ and $\beta$ curves intersect transversely in $\Sigma$, the associated tori intersect transversely in $\operatorname{Sym}^{g+|L|-1}(\Sigma)$. Let $\widehat{\mathrm{CFL}}(L)$ be the free abelian group generated by the intersection points $\mathbb{T}_{\alpha} \cap \mathbb{T}_{\beta}$. There is a boundary map on $\widehat{\mathrm{CFL}}(L)$ which gives it the structure of a chain complex, but we defer this until after a discussion of the multi-grading.

Supose that $\mathbf{x}$ and $\mathbf{y}$ are intersection points of $\mathbb{T}_{\alpha} \cap \mathbb{T}_{\beta}$, and let $\gamma_{+}$(respectively, $\gamma_{-}$) be the arc of the unit circle with positive (negative) real component. We denote by $\pi_{2}(\mathbf{x}, \mathbf{y})$ the set of all homotopy classes of maps from the unit disc in $\mathbb{C}$ to $\operatorname{Sym}^{g+|L|-1}(\Sigma)$ that satisfy the following:

$$
\left\{\begin{array}{c}
\phi(i)=\mathbf{x} \\
\phi(-i)=\mathbf{y} \\
\phi\left(\gamma_{+}\right) \subset \mathbb{T}_{\beta} \\
\phi\left(\gamma_{-}\right) \subset \mathbb{T}_{\alpha}
\end{array}\right\}
$$

If $\phi$ is such a map, we denote its image in the symmetric product by $\phi$ as well.

Definition 6 If $p$ is a point in $\Sigma-\left\{\alpha_{i}\right\}-\left\{\beta_{i}\right\}$, the intersection number of $\phi$ with $p$ is given by

$$
n_{p}(\phi)=\#\left(\phi \cap\left(\{p\} \times \operatorname{Sym}^{g+|L|-2}(\Sigma)\right)\right),
$$

where the intersection takes place in $\operatorname{Sym}^{g+|L|-1}(\Sigma)$.

\subsection{Domains and the filtration}

Since $\operatorname{Sym}^{g}(\Sigma)$ is difficult to visualize when $g>1$, it is convenient to work in the Heegaard surface itself as much as possible. Thus, we note the natural bijection between points of $\mathbb{T}_{\alpha} \cap \mathbb{T}_{\beta}$ and unordered $g$-tuples of intersection points on $\Sigma$ with the property that each of the attaching circles contributes to exactly one intersection point. The boundary of $\phi$ corresponds to a collection of arcs in the $\alpha$ and $\beta$ curves whose endpoints are these intersection points. 
Let $\left\{\Omega_{i}\right\}$ be the components of $\Sigma-\left\{\alpha_{i}\right\}-\left\{\beta_{i}\right\}$, and let $p_{i} \in \Omega_{i}$ be a point in each component.

Definition 7 If $\phi \in \pi_{2}(\mathbf{x}, \mathbf{y})$, the domain of $\phi$ is the formal sum

$$
D(\phi)=\sum_{i=1}^{N} n_{p_{i}}(\phi) \Omega_{i}
$$

One can construct $D(\phi)$ on $\Sigma$ as follows:

Let $\left\{x_{1}, x_{2}, \ldots x_{g}\right\}$ and $\left\{y_{1}, y_{2}, \ldots y_{g}\right\}$ be $g$-tuples of intersection points representing generators $\mathbf{x}$ and $\mathbf{y}$. Connect the $x_{i}$ to the $y_{j}$ along arcs of the attaching circles connecting the points, alternating between $\alpha$ and $\beta$ arcs. Additional copies of the $\alpha$ or $\beta$ circles may be required in order to get a null-homologous boundary. The null-homology determines the multiplicity of each of the $\Omega_{i}$, and gluing these regions along their boundary $\alpha$ and $\beta$ arcs builds an immersed surface representing an arbitrary $\phi \in \pi_{2}(\mathbf{x}, \mathbf{y})$.

Constructing a domain corresponding to a specific $\phi \in \pi_{2}(\mathbf{x}, \mathbf{y})$ demands more information about the space of discs.

Definition 8 A periodic domain $\theta$ is a linear combination of $\Omega_{i}$ with boundary a linear combination of $\left\{\alpha_{i}\right\} \cup\left\{\beta_{i}\right\}$ such that $n_{w_{i}}(\theta)=0$ for all $i$.

Two domains connecting the same generators may differ by a copy of $\Sigma$ or by a connected component of $\Sigma-\left\{\alpha_{i}\right\}$ or $\Sigma-\left\{\beta_{i}\right\}$. If $\phi$ and $\phi^{\prime}$ in $\pi_{2}(\mathbf{x}, \mathbf{y})$ satisfy $n_{\mathbf{w}}(\phi)=n_{\mathbf{w}}\left(\phi^{\prime}\right)$, then $D(\phi)$ and $D\left(\phi^{\prime}\right)$ differ by some periodic domain.

We are now in a position to define the multi-grading on $\widehat{\mathrm{CFL}}(L)$.

Definition 9 Let $\phi$ be a disc connecting $\mathbf{x}$ to $\mathbf{y}$, and let $F(\mathbf{x}, \mathbf{y})$ be the vector given by

$$
F(\mathbf{x}, \mathbf{y})=\left(n_{z_{1}}(\phi)-n_{w_{1}}(\phi), n_{z_{2}}(\phi)-n_{w_{2}}(\phi), \ldots n_{z_{|L|}}(\phi)-n_{w_{|L|}}(\phi)\right) .
$$

We say that $F(\mathbf{x}, \mathbf{y})$ is the filtration level of $\mathbf{x}$ relative to $\mathbf{y}$. This vector is independent of the choice of $\phi \in \pi_{2}(\mathbf{x}, \mathbf{y})$.

$F$ partitions the generators of $\widehat{\mathrm{CFL}}(L)$ into relative filtration levels: $\mathbf{x} \backsim \mathbf{y}$ if $F(\mathbf{x}, \mathbf{y})=$ $(0,0)$. The filtration support of link homology is naturally symmetric around a center point, and placing this point at the origin establishes absolute, rather than relative, filtration levels. Writing $\widehat{\mathrm{HFL}}(L, \mathbf{h})$ for the homology at the lattice point with coordinates $\mathbf{h}$, we have the symmetry relation

$$
\widehat{\mathrm{HFL}}(L, \mathbf{h}) \cong \widehat{\mathrm{HFL}}(L,-\mathbf{h}) .
$$


The meridians of the link components generate $H_{1}\left(S^{3}-L\right)$, and in fact the quantity $n_{z_{i}}(\phi)-n_{w_{i}}(\phi)$ may be interpreted as the linking number of the $i^{\text {th }}$ component of $L$ and a collection of loops in $S^{3}-L$ associated to the generators $\mathbf{x}$ and $\mathbf{y}$. (See Ozsváth and Szabó [5, Section 3.6] for this construction.) This in turn allows each filtration level $\mathbf{h}$ to be identified with an element of $H_{1}\left(S^{3}-L\right)$. For $\mathbf{x} \in \widehat{\mathrm{CFL}}(L, \mathbf{h})$ we refer to the $H_{1}\left(S^{3}-L\right)$ grading $\mathbf{h}$ as the filtration level of $\mathbf{x}$ in order to distinguish this from the Maslov, or homological, grading, which we define in the next section. The boundary map on $\widehat{\mathrm{CFL}}(L)$ counts discs connecting pairs of generators, and it preserves the filtration level while lowering the homological grading by one.

\subsection{The homological grading}

A holomorphic structure on $\Sigma$ lifts to a holomorphic structure on $\operatorname{Sym}^{g+|L|-1}(\Sigma)$, allowing one to study the moduli space of holomorphic representatives of a homotopy class in $\pi_{2}(\mathbf{x}, \mathbf{y})$. For a given $\phi \in \pi_{2}(\mathbf{x}, \mathbf{y})$, the Maslov index gives the expected dimension of the moduli space $\mathcal{M}(\phi)$ of holomorphic representatives of $\phi$ (see Ozsvath and Szabó [8]). Additionally, the Maslov index equips the generators of $\widehat{\mathrm{CFL}}(L)$ with a relative $\mathbb{Z}$ grading which can be computed combinatorially from the domain $D(\phi)$ in the Heegaard diagram (see Lipshitz [2]).

Let $\mathbf{x}=\left(x_{1}, x_{2}, \ldots x_{g}\right)$ and $\mathbf{y}=\left(y_{1}, y_{2}, \ldots y_{g}\right)$ be generators of $\widehat{\mathrm{CFL}}(L)$, and write $\bar{n}_{x_{i}}$ (respectively, $\bar{n}_{y_{i}}$ ) for the local multiplicity of the intersection point $x_{i}\left(y_{i}\right)$. Letting $e(D(\phi))$ denote the Euler measure of a domain $D(\phi)$ for $\phi \in \pi_{2}(\mathbf{x}, \mathbf{y})$, the relative grading of $\mathbf{x}$ and $\mathbf{y}$ is given by the integer $\mu(\phi)$ :

$$
\left.\mu(\phi)=e(D(\phi))+\sum_{i} \bar{n}_{x_{i}}+\sum_{i} \bar{n}_{y_{i}}-2 \sum_{i=1}^{|L|} n_{w_{i}}(\phi)\right) .
$$

If every $\Omega_{i}$ in $D(\phi)$ has multiplicity zero or one, this can be expressed even more concretely. Consider $D(\phi)$ as a subsurface in $\Sigma$ with boundary in the four-valent graph formed by the $\alpha$ and $\beta$ curves. Let $k$ be the number of corner points where $D(\phi)$ fills a single quadrant, and let $l$ be the number of corners where it fills three quadrants. Then

$$
\left.\mu(\phi)=\chi(D(\phi))-\frac{k}{4}+\frac{l}{4}+\sum_{i} \bar{n}_{x_{i}}+\sum_{i} \bar{n}_{y_{i}}-2 n_{w}(D(\phi))\right) .
$$

We will be particularly interested in the case where $\mu(\phi)=1$.

One may treat the marked points on the boundary of the complex unit disc as punctures, and the unit disc with twice-punctured boundary is conformally equivalent to a vertical 
strip in the complex plane. $\mathbb{R}$ acts naturally on this strip via vertical translation, and taking the quotient with respect to this action decreases the dimension of the space of maps by one. We denote this reduced moduli space by $\mathcal{M}(\phi) / \mathbb{R}$. For a generic complex structure, $\mu(\phi)=1$ implies that $\mathcal{M}(\phi) / \mathbb{R}$ is zero-dimensional. This permits the following definition:

Definition 10 The boundary map $\widehat{\partial}$ on $\widehat{\mathrm{CFL}}(L)$ is given by

$$
\widehat{\partial}(\mathbf{x})=\sum_{\mathbf{y}} \sum_{\substack{\phi \in \pi_{2}(\mathbf{x}, \mathbf{y}): \mu(\phi)=1, n_{\mathbf{w}}(\phi)=n_{\mathbf{z}}(\phi)=0}} \#(\mathcal{M}(\phi) / \mathbb{R}) \mathbf{y}
$$

This map satisfies $\widehat{\partial}^{2}=0$, so $(\widehat{\mathrm{CFL}}, \widehat{\partial})$ is a chain complex whose homology is the link invariant $\widehat{\mathrm{HFL}}(L)$. Because the boundary preserves the filtration level while lowering the homological grading by one, the homology decomposes as the direct sum

$$
\widehat{\operatorname{HFL}}(L)=\bigoplus_{\mathbf{h} \in H_{1}\left(S^{3}-L, \mathbb{Z}\right)} \widehat{\operatorname{HFL}}(L, \mathbf{h})
$$

Recall from Section 2 that the dual Thurston norm is defined on $H_{2}\left(S^{3}, L\right)^{*} \cong$ $H_{1}\left(S^{3}-L\right)$. Thus one may compare the dual norm ball to the filtration support of $\widehat{\mathrm{HFL}}(L)$, the set $\{\mathbf{h}: \widehat{\mathrm{HFL}}(L, \mathbf{h}) \neq 0\})$.

Theorem 2 (Ozsváth-Szabó [6]) Let $\left\{\mu_{i}\right\}_{i=1}^{|L|}$ be the meridians of an oriented link $L$ that has no trivial components. For each $\mathbf{k} \in H^{1}\left(S^{3}-L\right)$

$$
\|P D(\mathbf{k})\|_{T}+\sum_{i=1}^{|L|}\left\langle\boldsymbol{k}, \mu_{i}\right\rangle=2\left(\max _{\boldsymbol{h} \in H_{1}(L, \mathbb{Z}): \widehat{\operatorname{HFL}}(L, h) \neq 0}|\langle\boldsymbol{k}, \boldsymbol{h}\rangle|\right) .
$$

Thus, the setwise sum in $\mathbb{R}^{|L|}$ of the dual Thurston polytope and the cube of edge-length two is twice the convex hull of the filtration support of $\widehat{\mathrm{HFL}}(L)$.

\section{Proof of Theorem 1}

\section{1 $\widehat{\mathrm{HFL}}(K)$}

We begin by computing the dual Thurston polytope for the knotted component $K$ of $P_{q_{1}, r_{1}, q_{2}, r_{2}}$. Since $K$ is a one-component link, this is simply twice the Thurston norm of the homology class of any Seifert surface for $K$. In addition to serving as a toy calculation illustrating the techniques, the result will prove useful in the sequent. 
$K$ is the connected sum of the torus knots $T\left(-2,2 r_{1}+1\right)$ and $T\left(2,2 r_{2}+1\right)$; Figure 10 shows an example of a Heegaard diagram compatible with $K$. From this diagram it is clear that each generator of $\widehat{\mathrm{CFL}}(K)$ corresponds to a pair of intersection points $a_{*} \in \alpha_{1} \cap \beta_{1}$ and $A_{*} \in \alpha_{2} \cap \beta_{2}$. Holding the $\alpha_{2} \cap \beta_{2}$ intersection point constant, one determines the relative filtration levels of the $a_{i}$ by finding a domain connecting any pair. Relative filtration is additive under concatenation of discs, so it suffices to show that successively numbered intersection points have relative filtration one. The same holds for the points of $\alpha_{2} \cap \beta_{2}$, so the filtration level of the generator $\left(a_{2 r_{1}}, A_{2 r_{2}}\right)$ is $2 r_{1}+2 r_{2}$ greater than that of $\left(a_{0}, A_{0}\right)$.

The two generators $\left(a_{2 r_{1}}, A_{2 r_{2}}\right)$ and $\left(a_{0}, A_{0}\right)$ are unique in their respective filtration levels, so each represents a non-trivial homology class. The Heegaard Floer polytope is therefore an interval of length $2 r_{1}+2 r_{2}$, and applying the symmetry relation of Equation (5-2) identifies it with $\left[-r_{1}-r_{2}, r_{1}+r_{2}\right]$ in $\mathbb{Z} \cong H_{1}\left(S^{3}-K\right)$. Theorem 2 translates this into information about the Thurston norm of $S^{3}-K$; doubling the interval and subtracting one reveals that the minimal complexity of a Seifert surface for $K$ is $2 r_{1}+2 r_{2}-1$. If $K$ is drawn as the pretzel knot $P\left(2 r_{1}+1,0,-2 r_{2}-1\right)$, Seifert's algorithm produces a surface realizing the Thurston norm.

\subsection{The filtration on $\widehat{\mathrm{CFL}}\left(P_{q_{1}, r_{1}, q_{2}, r_{2}}\right)$}

Turning now to the proof of the main theorem, we use a Heegaard diagram of the type indicated in Figure 11 to study the Heegaard Floer link homology of $P\left(-2 r_{1}-\right.$ $\left.1,2 q_{1},-2 q_{2}, 2 r_{2}+1\right)$.

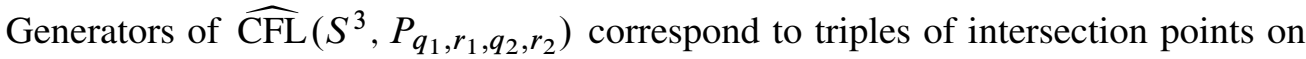
the diagram, and these split naturally into two types. Denote triples of the form $\left(\alpha_{1} \cap \beta_{1}, \alpha_{2} \cap \beta_{3}, \alpha_{3} \cap \beta_{2}\right)$ by lowercase letters $\left(a_{*}, b_{*}, c_{*}\right)$, and triples of the form $\left(\alpha_{1} \cap \beta_{2}, \alpha_{2} \cap \beta_{1}, \alpha_{3} \cap \beta_{3}\right)$ by uppercase letters $\left(A_{*}, B_{*}, C_{*}\right)$.

Lemma 1 The filtration levels of $\left(A_{i}, B_{j}, C_{k}\right)$ and $\left(a_{i}, b_{j}, c_{k}\right)$ are equal.

Proof of Lemma 1 Although Equation (5-2) determines the absolute filtration on $\widehat{\mathrm{HFL}}(L)$, at the chain level only relative filtration is computable. We will therefore assign coordinates pro tem to identify $H_{1}\left(S^{3}-P_{q_{1}, r_{1}, q_{2}, r_{2}}\right)$ and $\mathbb{Z}^{|L|}$, with the understanding that some translation may be required at the level of homology in order to satisfy the symmetry requirement. Thus we identify the filtration level of the generator $\left(a_{1}, b_{1}, c_{1}\right)$ with the lattice point $(0,1)$.

The filtration level of any lowercase generator is the sum of contributions from each of the constituent intersection points, and varying a single intersection point at a time 


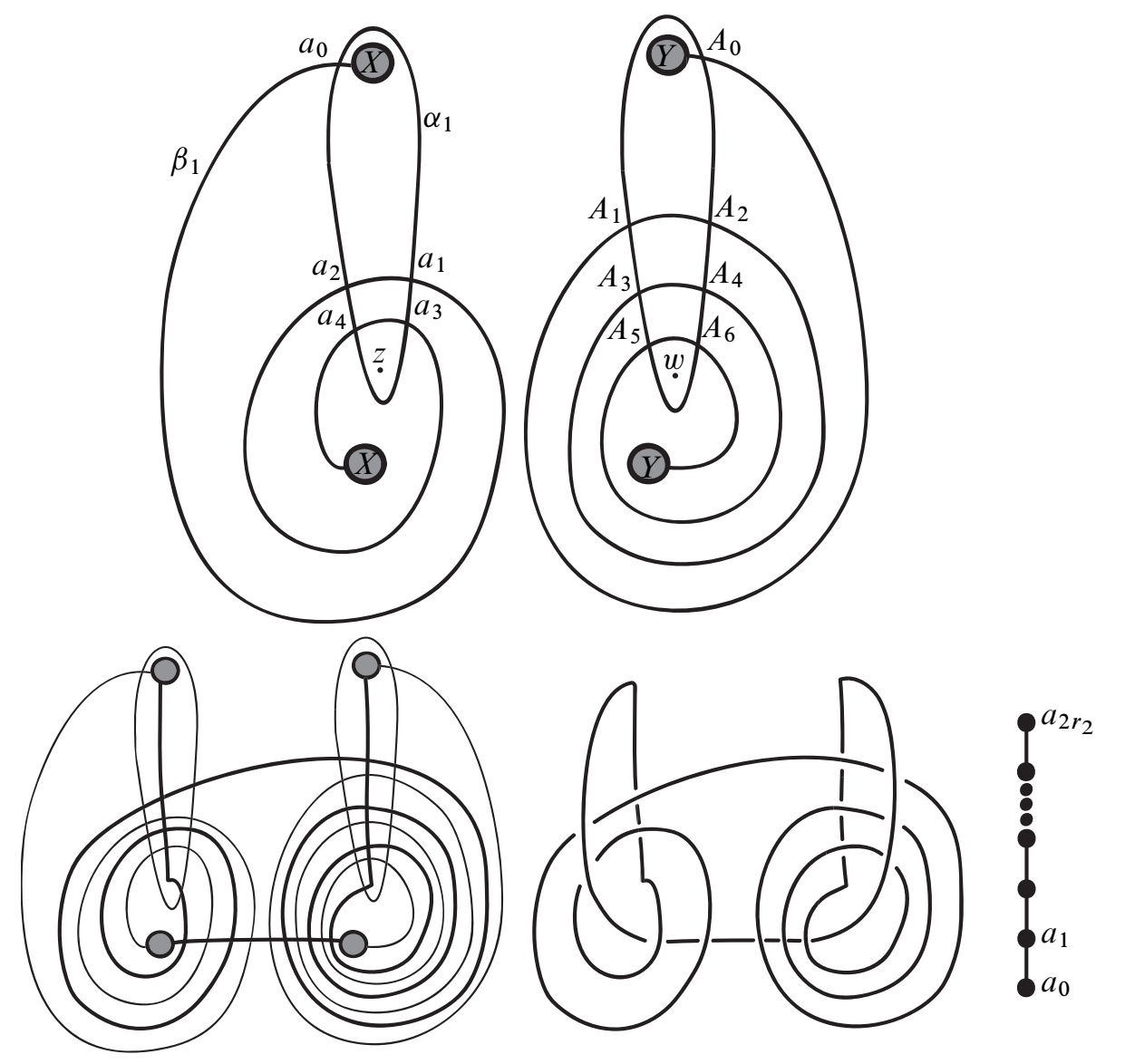

Figure 10: Top: A genus-two Heegaard diagram compatible with $T(-2,5) \# T(2,7)$. The vertical pairs of shaded circles indicate one-handles. Bottom: One may recover the knot from the diagram by connecting $z$ to $w$ via a pair of arcs, each avoiding one of $\left\{\alpha_{i}\right\}$ or $\left\{\beta_{i}\right\}$, and pushing these arcs into the appropriate handlebodies. The lower right figure indicates that $F\left(a_{i}, a_{i-1}\right)=1$, and the same relationship holds for the uppercase generators $A_{*}$.

allows us to determine the relative filtrations of the intersection points between a fixed $\alpha_{i}$ and $\beta_{j}$. This data is indicated for each of the lowercase intersection points in the top diagram in Figure 12. We stipulate further that the points $b_{1}$ and $c_{1}$ contribute $(0,0)$ to the filtration level, and this implies that the contribution of $a_{1}$ is $(0,1)$. Thus, the relative filtration graphs are fixed in $\mathbb{Z}^{|L|}$, and the filtration level of any lowercase generator is the sum of the coordinates corresponding to each of its three intersection points. (See the lower diagram in Figure 12.) This establishes filtration levels for each 

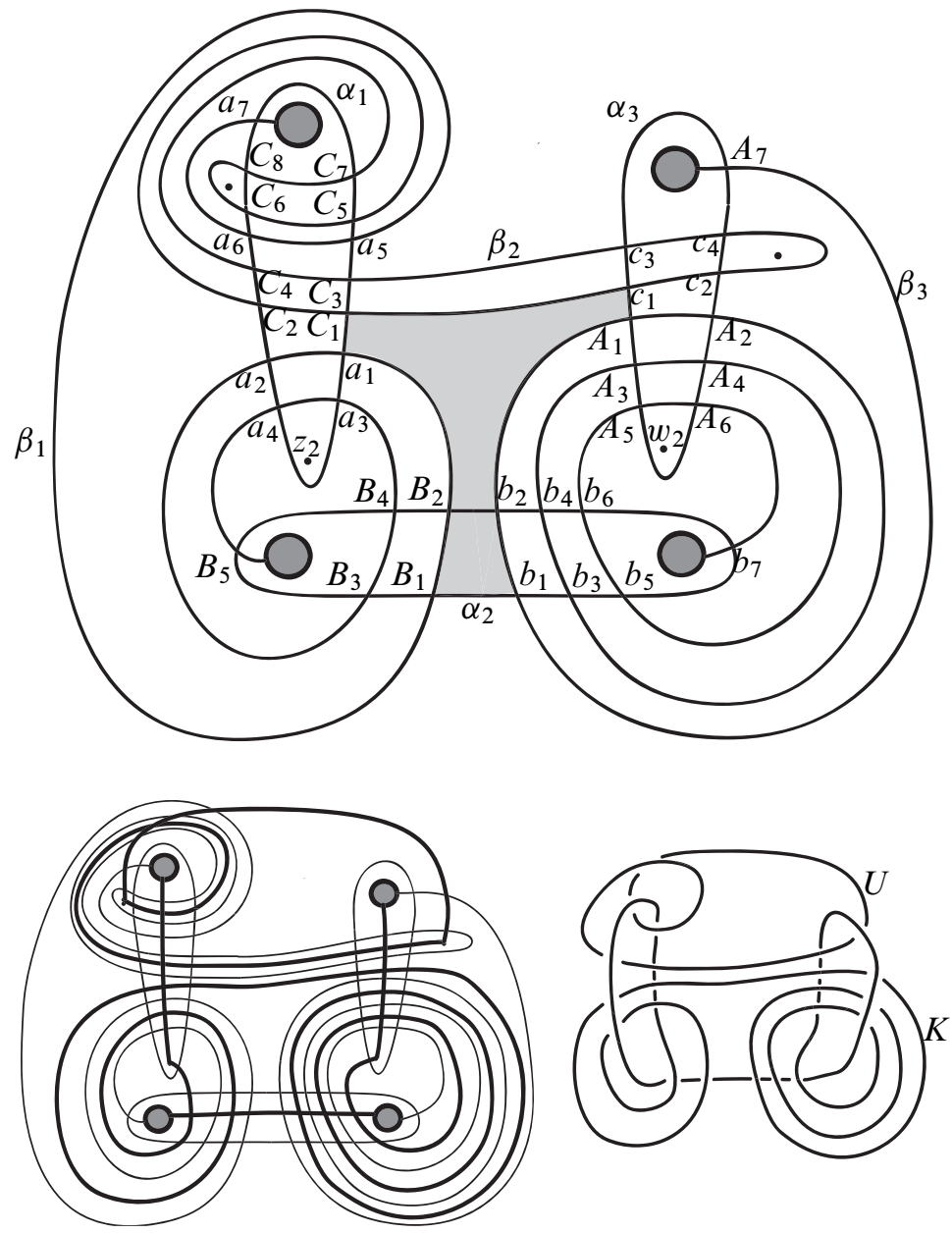

Figure 11: Top: A genus two Heegaard diagram for $P_{2,2,1,3}$. The shaded region is the domain connecting the generators $\left(a_{1}, b_{1}, c_{1}\right)$ and $\left(A_{1}, B_{1}, C_{1}\right)$ which is used in the proof of Lemma 1. Bottom: One may recover $L$ from this diagram by connecting each $\left(z_{i}, w_{i}\right)$ pair by an arc in the complement of the $\left\{\alpha_{i}\right\}$ and an arc in the complement of the $\left\{\beta_{i}\right\}$ and pushing the arcs into the appropriate handlebodies.

of the lowercase generators, and for any pair of generators, the difference between these is exactly the relative filtration computed from the domain of a disc connecting them.

Algebraic $6 \mathcal{G}$ Geometric $\mathcal{T}$ opology, Volume 8 (2008) 


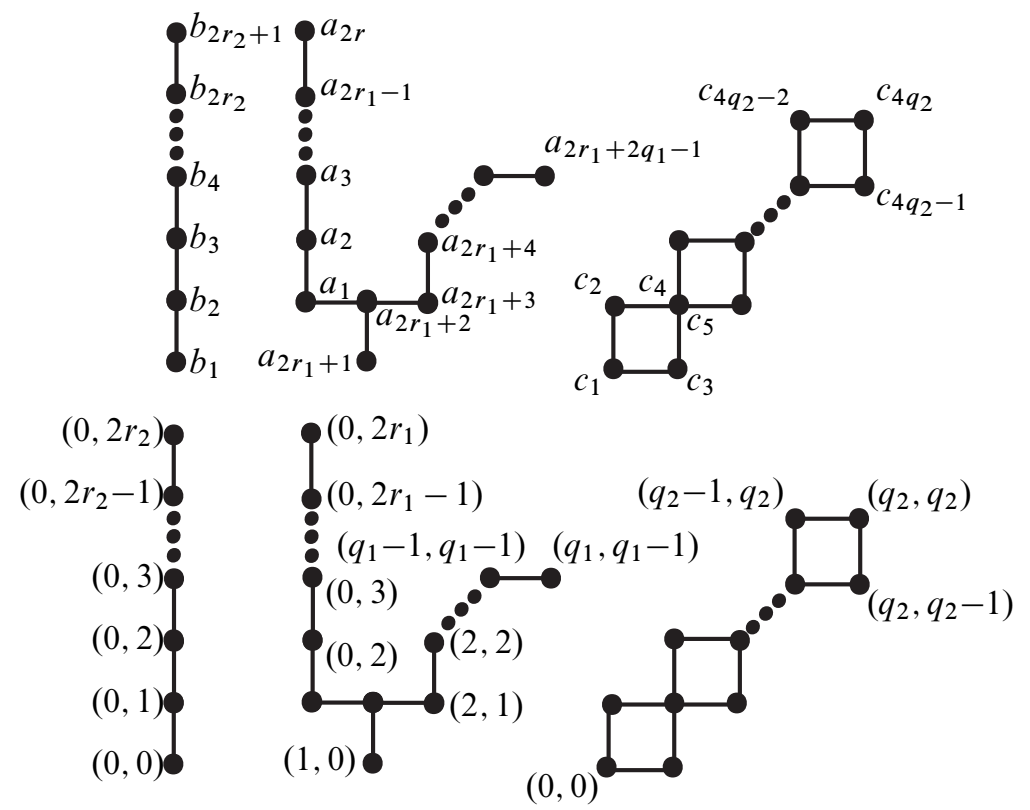

Figure 12: In the first diagram we show the relative filtrations of each type of lowercase intersection point, and in the second diagram we assign temporary coordinates. Switching the subscripts 1 and 2 gives the filtration data for the uppercase generators.

There is a hexagon disjoint from all the basepoints which connects $\left(A_{1}, B_{1}, C_{1}\right)$ to $\left(a_{1}, b_{1}, c_{1}\right)$, so these generators are in the same filtration level. (See Figure 11.) Now suppose that $D(\phi)$ is a domain connecting $\left(a_{1}, b_{1}, c_{1}\right)$ to another lowercase generator $\left(a_{i}, b_{j}, c_{k}\right)$ with filtration $F(\phi)=\left(n_{z_{1}}(\phi)-n_{w_{1}}(\phi), n_{z_{2}}(\phi)-n_{w_{2}}(\phi)\right)$. The mirror image of $D(\phi)$ is a domain $D\left(\phi^{\prime}\right)$ connecting $\left(A_{i}, B_{j}, C_{k}\right)$ to $\left(A_{1}, B_{1}, C_{1}\right)$ and satisfying $F\left(\phi^{\prime}\right)=-F(\phi)$. Figure 13 shows an example of such a pair. Consequently each uppercase generator has the same filtration level as its lowercase counterpart, and the relative and coordinate filtration graphs for the uppercase generators are identical to those for the lowercase generators with the same indices. Note that if $q_{1} \neq q_{2}$ or $r_{1} \neq r_{2}$, some generators will not be part of such pairs.

Corollary 2 The multivariable Alexander polynomial vanishes for the links $P_{q, r}$.

Proof of Corollary 2 The graded Euler characteristic of Heegaard Floer link homology is the multivariable Alexander polynomial (see Ozsváth and Szabó [5]). According to Lemma 1, the generators of $\widehat{C F K}\left(S^{3}, P_{q, r}\right)$ appear in pairs in the same filtration level. 


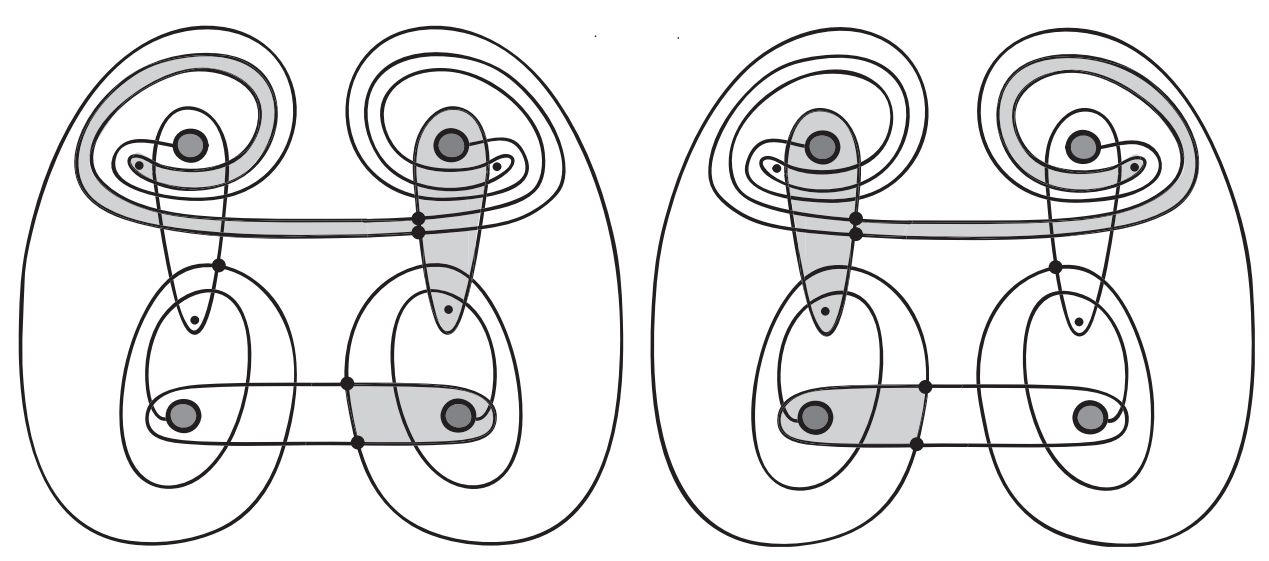

Figure 13: The shaded area on the left indicates a domain connecting $\left(a_{1}, b_{1}, c_{1}\right)$ to $\left(a_{1}, b_{2}, c_{3}\right)$, whereas the mirror-image domain on the righthand diagram indicates a domain connecting $\left(A_{1}, B_{2}, C_{3}\right)$ to $\left(A_{1}, B_{1}, C_{1}\right)$.

We claim that the gradings of such a pair of elements are always of opposite parity. In this case, an application of the Euler-Poincare principle shows that the graded Euler characteristic of the homology vanishes.

To prove the claim, we first note that it holds for the pair $\left(a_{1}, b_{1}, c_{1}\right)$ and $\left(A_{1}, B_{1}, C_{1}\right)$, since the hexagonal domain cited above has Maslov index one. Furthermore, if $D(\phi)$ and $D\left(\phi^{\prime}\right)$ are the mirror-image domains from the proof of Lemma 1, then the relative gradings of the corresponding generator pairs are equal mod two; the only difference comes from the even-integer-valued terms $2 n_{w_{i}}$.

\subsection{Proof of Theorem 1}

According to Theorem 2, $B_{T}^{*}\left(P_{q_{1}, r_{1}, q_{2}, r_{2}}\right)$ is determined by the convex hull of the filtration support of $\widehat{\operatorname{HFL}}\left(S^{3}, P_{q_{1}, r_{1}, q_{2}, r_{2}}\right)$. We thus need to establish which filtration levels have non-trivial homology. A filtration level must have at least a single generator to support nontrivial homology, so we have a priori upper bounds on the dimensions of the Thurston polytope which come from the filtration support of $\widehat{\mathrm{CFL}}\left(S^{3}, L\right)$ :

Definition 11 Let $S$ denote the set $\left\{(x, y)\right.$ : $\left.\left.\widehat{\mathrm{CFL}}\left(S^{3}, P_{q_{1}, r_{1}, q_{2}, r_{2}},(x, y)\right) \neq \varnothing\right)\right\}$, and let $\mathfrak{H}(S)$ be the convex hull of $S$.

Throughout the proof we will use the coordinates assigned in the proof of Lemma 1. 


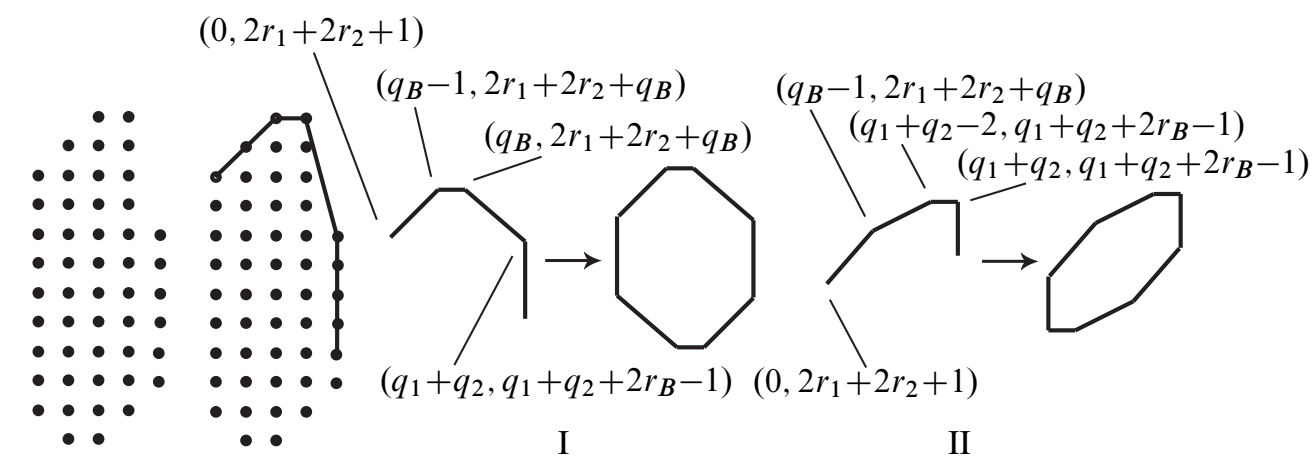

Figure 14: From the left: $S$ for $P_{2,2}$. The second figure indicates vertices of $\mathfrak{H}(S)$ which are also in $\mathfrak{H}(\widehat{\mathrm{HFL}})$. The remaining pairs indicate how the entire polytope may be constructed from half its faces, and the coordinates shown are established in the proof of Theorem 1. The central pair (type I) corresponds to the case $2 r_{S}>q_{S}-1$, and the pair of figures on the right (type II) corresponds to the case $2 r_{S}<q_{S}-1$.

In an ideal world, the filtration support of the chain complex and the nontrivial homology might be equal and the polytopes $\mathfrak{H}(S)$ and $\mathfrak{H}(\widehat{\mathrm{HFL}})$ would coincide. This is obviously false, however, as the former is not symmetric. Lowering our expectations a bit, we can ask if the two polytopes share enough of the same vertices to ensure that the symmetry relation determines the rest of of $\mathfrak{H}(\widehat{\mathrm{HFL}})$. This general approach is indicated in Figure 14. We first compute $S$ in order to get bounds on the Heegaard Floer polytope, and then we study the subcomplexes at the extremal vertices of $S$ to see which support nontrivial homology. Half the faces of a symmetric polytope suffice to determine the entire object, so we can try to pick a subset of the extremal vertices where the Heegaard Floer homology will be easier to analyze. The shape of the resulting polytope depends on the relative values of the $q_{i}$ and $r_{i}$.

The proof proceeds in two steps, each of which establishes certain vertices of $\mathfrak{H}(\widehat{\mathrm{HFL}})$. For ease of reference, we will distinguish between links of type I $\left(2 r_{S}>q_{S}-1\right)$ and II $\left(2 r_{S}<q_{S}-1\right)$ as indicated in Figure 14. If $2 r_{S}=q_{S}-1$, the proof is as in the type II case. Lemma 2 uses direct computation to show that three lattice points in $S$ also support nontrivial homology, and Lemma 3 determines the length of the right vertical edge of $\mathfrak{H}(\widehat{\mathrm{HFL}})$.

\section{Proof of Theorem 1}

Lemma 2 Let $(x, y)$ be any of the lattice points

$$
\left\{\left(0,2 r_{1}+2 r_{2}+1\right), \quad\left(q_{B}-1,2 r_{1}+2 r_{2}+q_{B}\right), \quad\left(q_{B}, 2 r_{1}+2 r_{2}+q_{B}\right)\right\} .
$$


The Heegaard Floer groups

$$
\widehat{\operatorname{HFL}}\left(S^{3}, P_{q_{1}, r_{1}, q_{2}, r_{2}},(x, y)\right)
$$

are nontrivial.

The first two lattice points of Lemma 2 are always vertices of $\mathfrak{H}(S)$. Since they support nontrivial homology, they also define vertices of the Heegaard Floer polytope $\mathfrak{H}(\widehat{\mathrm{HFL}})$. This shows that $\mathfrak{H}(\widehat{\mathrm{HFL}})$ and $\mathfrak{H}(S)$ share a face with slope one. In the type I case, the third point is also a vertex of $\mathfrak{H}(S)$, and the two polytopes share a side of slope zero as well.

When $r_{1} \neq r_{2}$, the filtration levels $\left(q_{1}+q_{2}-2, q_{1}+q_{2}+2 r_{B}-1\right)$ and $\left(q_{1}+q_{2}, q_{1}+\right.$ $\left.q_{2}+2 r_{B}-1\right)$ each support a unique generator and thus have nontrivial homology. These are vertices of $\mathfrak{H}(S)$, and studying the extreme points of $S$ for $q_{B} \leq x \leq q_{1}+q_{2}$ reveals that these vertices suffice to determine the slope of all the faces. Specifically, in the type I case $\mathfrak{H}(\widehat{\mathrm{HFL}})$ shares an edge with $\mathfrak{H}(S)$ connecting the lattice points $\left(q_{B}, 2 r_{1}+2 r_{2}+q_{B}\right)$ and $\left(q_{1}+q_{2}, q_{1}+q_{2}+2 r_{B}-1\right)$. In the type II case, $\mathfrak{H}(\widehat{\mathrm{HFL}})$ shares edges with $\mathfrak{H}(S)$ which connect $\left(q_{B}-1,2 r_{1}+2 r_{2}+q_{B}\right)$ to $\left(q_{1}+q_{2}-2, q_{1}+\right.$ $\left.q_{2}+2 r_{B}-1\right)$ and $\left(q_{1}+q_{2}-2, q_{1}+q_{2}+2 r_{B}-1\right)$ to $\left(q_{1}+q_{2}, q_{1}+q_{2}+2 r_{B}-1\right)$. Geometrically, this means that the two polytopes agree along all their top faces. These lattice points are labeled in Figure 14, and it may be helpful to refer to the diagram.

To completely determine $\mathfrak{H}\left(\widehat{\mathrm{HFL}}\left(P_{q_{1}, r_{1}, q_{2}, r_{2}}\right)\right)$ we need also the length of the vertical sides, but here the two polytopes differ: the two generators in filtration level $\left(q_{1}+\right.$ $\left.q_{2}, q_{1}+q_{2}-2\right)$ are connected by a simply-connected domain which has a holomorphic representative, so the homology is trivial. (This is shown explicitly in the proof of Lemma 3.)

Lemma 3 The length of each of the vertical edges of $\mathfrak{H}\left(\widehat{\operatorname{HFL}}\left(P_{q_{1}, r_{1}, q_{2}, r_{2}}\right)\right)$ is $2 r_{B}$.

Since the right vertical edge of $\mathfrak{H}(S)$ has length $2 r_{B}+1$ and we have already identified the lattice point $\left(q_{1}+q_{2}, q_{1}+q_{2}-2\right)$ as supporting trivial homology, this determines the Heegaard Floer polytope for type I links and for type II links when $r_{1} \neq r_{2}$. The coordinates stated in the theorem then follow from first replacing the temporary filtration levels adopted in Lemma 1 with those dictated by Equation (5-2) and then applying Theorem 2. In order to complete the final case (type II, $r_{1}=r_{2}$ ), we need to show that the homology at $\left(q_{1}+q_{2}-2, q_{1}+q_{2}+2 r_{B}-1\right)$ is nontrivial when the filtration level supports two generators. We include this argument after the proof of Lemma 3. 
Proof of Lemma 2 If $q_{1} \neq q_{2}$, the filtration levels $\left(q_{B}-1,2 r_{1}+2 r_{2}+q_{B}\right)$ and $\left(q_{B}, 2 r_{1}+2 r_{2}+q_{B}\right)$ each have a unique generator, so the homology is also onedimensional. If $q_{1}=q_{2}$, there is a pair of generators at each of these filtration levels and also at $\left(0,2 r_{1}+2 r_{2}+1\right)$. We prove that $\widehat{\operatorname{HFL}}\left(S^{3}, P_{q_{1}, r_{1}, q_{2}, r_{2}},(x, y)\right) \neq 0$ by showing that for each of these pairs, no disc $\phi$ connecting them can have a holomorphic representative.

The argument is by linear algebra; by definition, a boundary disc satisfies $n_{z_{i}}(\phi)=$ $n_{w_{i}}(\phi)=0$ for all $i$, and a disc with a holomorphic representative must also satisfy $n_{p_{i}}(\phi) \geq 0$ for all points $p_{i}$ in the complement of the attaching circles (see Ozsváth and Szabó [8, Lemma 3.2]). For each of the generator pairs in question, we show there is no domain satisfying all these constraints.

First construct a domain for an arbitrary $\phi_{1}$ in

$$
\pi_{2}\left(\left(A_{2 r_{2}}, B_{2 r_{1}+1}, C_{2}\right),\left(a_{2 r_{1}}, b_{2 r_{2}+1}, c_{2}\right)\right)
$$

as indicated in Figure 15. The $\alpha$ and $\beta$ curves each separate the surface into two connected components, and these subsurfaces may be added or subtracted from $D\left(\phi_{1}\right)$ to change the basepoint multiplicities. Subtracting a copy of the component of $\Sigma-\left\{\alpha_{i}\right\}$ containing $z_{2}$ produces a new domain $D\left(\phi_{2}\right)$ satisfying $n_{w_{1}}\left(\phi_{2}\right)=$ $n_{z_{1}}\left(\phi_{2}\right)=n_{w_{2}}\left(\phi_{2}\right)=n_{z_{2}}\left(\phi_{2}\right)=0$. Note that the diagram has an extra marked point $p$ and that $n_{p}\left(\phi_{2}\right)<0$. Recall from Section 5 that any other domain with the same basepoint intersection numbers can differ from $D\left(\phi_{2}\right)$ only by periodic domains. The space of periodic domains on this diagram is one-dimensional over $\mathbb{Z}$, and the generating periodic domain has multiplicity zero at the extra marked point $p$. Thus, for any periodic domain $\theta, n_{p}\left(\phi_{2}+\theta\right)=n_{p}(\phi)$. The positivity and null-intersection conditions cannot be simultaneously realized, so the moduli space $\mathcal{M}(\phi) / \mathbb{R}$ is empty and $\widehat{\operatorname{HFL}}\left(P_{q_{1}, r_{1}, q_{2}, r_{2}},\left(0,2 r_{1}+2 r_{2}+1\right)\right)$ has rank two.

This illustrates a general technique which is often useful for studying the boundary map. If, for some $\phi \in \pi_{2}(\mathbf{x}, \mathbf{y})$, there is a point $p$ with $n_{p}(\theta)=0$ and either $n_{p}(\phi)<n_{w_{i}}(\phi)$ or $n_{p}(\phi)<n_{z_{i}}(\phi)$, then there can be no boundary disc connecting $\mathbf{x}$ to $\mathbf{y}$. Figure 16 shows domains and additional basepoints for the other two lattice points in the case that $q_{1}=q_{2}$.

We would like to show that the rank-four chain complex with filtration level $\left(q_{1}+\right.$ $\left.q_{2}, q_{1}+q_{2}-1\right)$ supports non-trivial homology. In the case that $r_{1} \neq r_{2}$, this would complete our computation of the Heegaard Floer polytope, and in the general case it would establish the penultimate vertex. However, the domains connecting these generators are complicated, and explicitly counting points in the reduced moduli space 


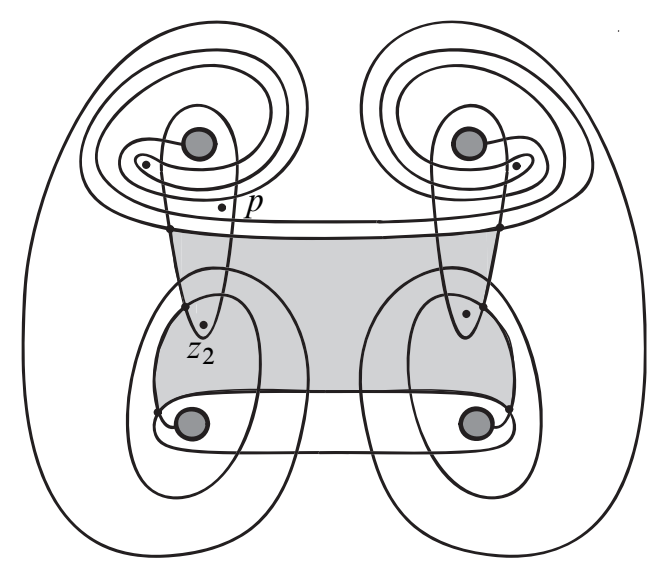

Figure 15: The figure shows $D(\phi)$ for a disc connecting the generator $\left(A_{2 r_{2}}, B_{2 r_{1}+1}, C_{2}\right)$ to $\left(a_{2 r_{1}}, b_{2 r_{2}+1}, c_{2}\right)$. Since $n_{z_{2}}(\phi)>n_{p}(\phi)$, $\widehat{\mathrm{HFL}}\left(L,\left(0,2 r_{1}+2 r_{2}+1\right) \neq 0\right.$.
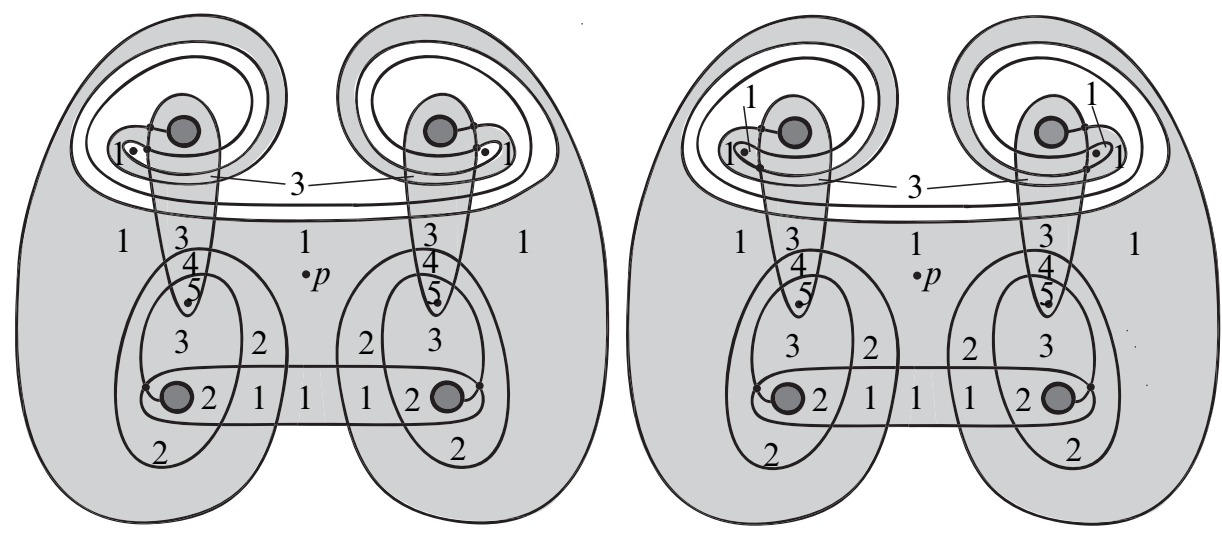

Figure 16: The domains shown here complete the proof of Lemma 2 for the case $q_{1}=q_{2}$. Components of $\Sigma-\left\{\alpha_{i}\right\}-\left\{\beta_{i}\right\}$ are labeled with their multiplicity in the given domains, and components which are not labeled have multiplicity two. Left: $\left(q_{B}, 2 r_{1}+2 r_{2}+q_{B}\right)$. Right: $\left(q_{B}-1,2 r_{1}+2 r_{2}+q_{B}\right)$.

is prohibitively difficult. Instead, we appeal to a new Heegaard diagram in order to prove Lemma 3.

\section{Proof of Lemma 3}

Algebraic ${ }^{3} \mathcal{G}$ Geometric Topology, Volume 8 (2008) 


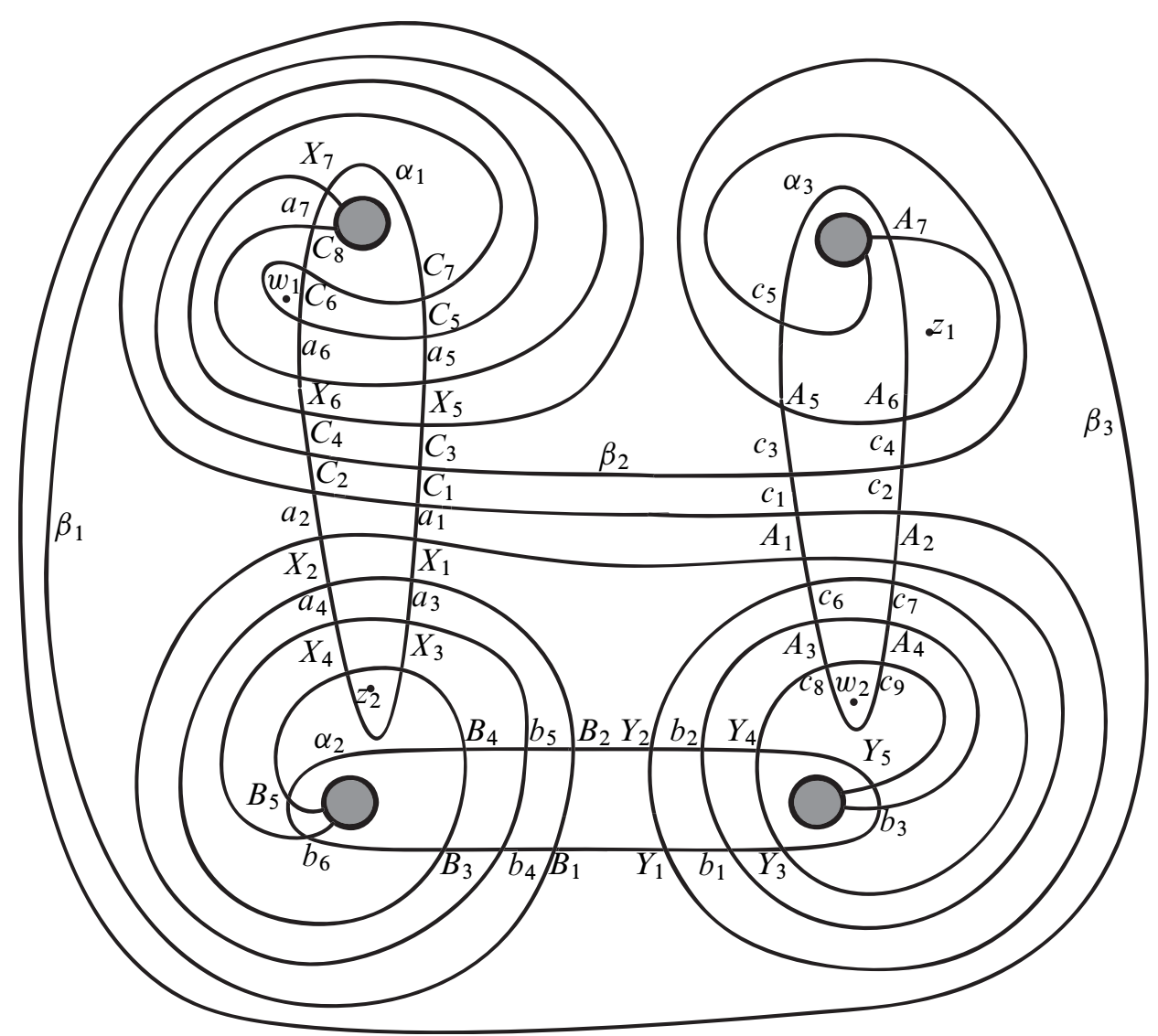

Figure 17: A Heegaard diagram for $P_{2,2}$. This diagram is related to the one of the type shown in Figure 11 by two handleslides.

Beginning with a Heegaard diagram of the type shown in Figure 11 for $P_{q_{1}, r, q_{2}, r}$, handleslide $\beta_{2}$ across $\beta_{3}$, and then handleslide $\beta_{3}$ across $\beta_{1}$. (See Figure 17 for an example of the new Heegaard diagram.) This creates intersections between previously disjoint curves; in addition to the intersection points of types $A, B, C, a, b$, and $c$, we have $X_{i}\left(\alpha_{1} \cap \beta_{3}\right)$ and $Y_{i}\left(\alpha_{2} \cap \beta_{2}\right)$. Altogether there are four types of generators: $\{a b c, A B C, a A Y, c B X\}$.

As before, we assign temporary coordinates consistent with the relative filtration levels of the various intersection points. These are indicated in Figure 18 and are chosen to agree with the coordinates of the first diagram. We achieve this consistency by comparing the filtration support of two different diagrams for a single link of type I with $r_{1} \neq r_{2}$. In the first diagram, the filtration levels $\left(0,2 r_{1}+2 r_{2}+1\right)$ and $\left(q_{1}+q_{2}, q_{1}+q_{2}+2 r_{B}-1\right)$ each support nontrivial homology (Lemma 2). This 


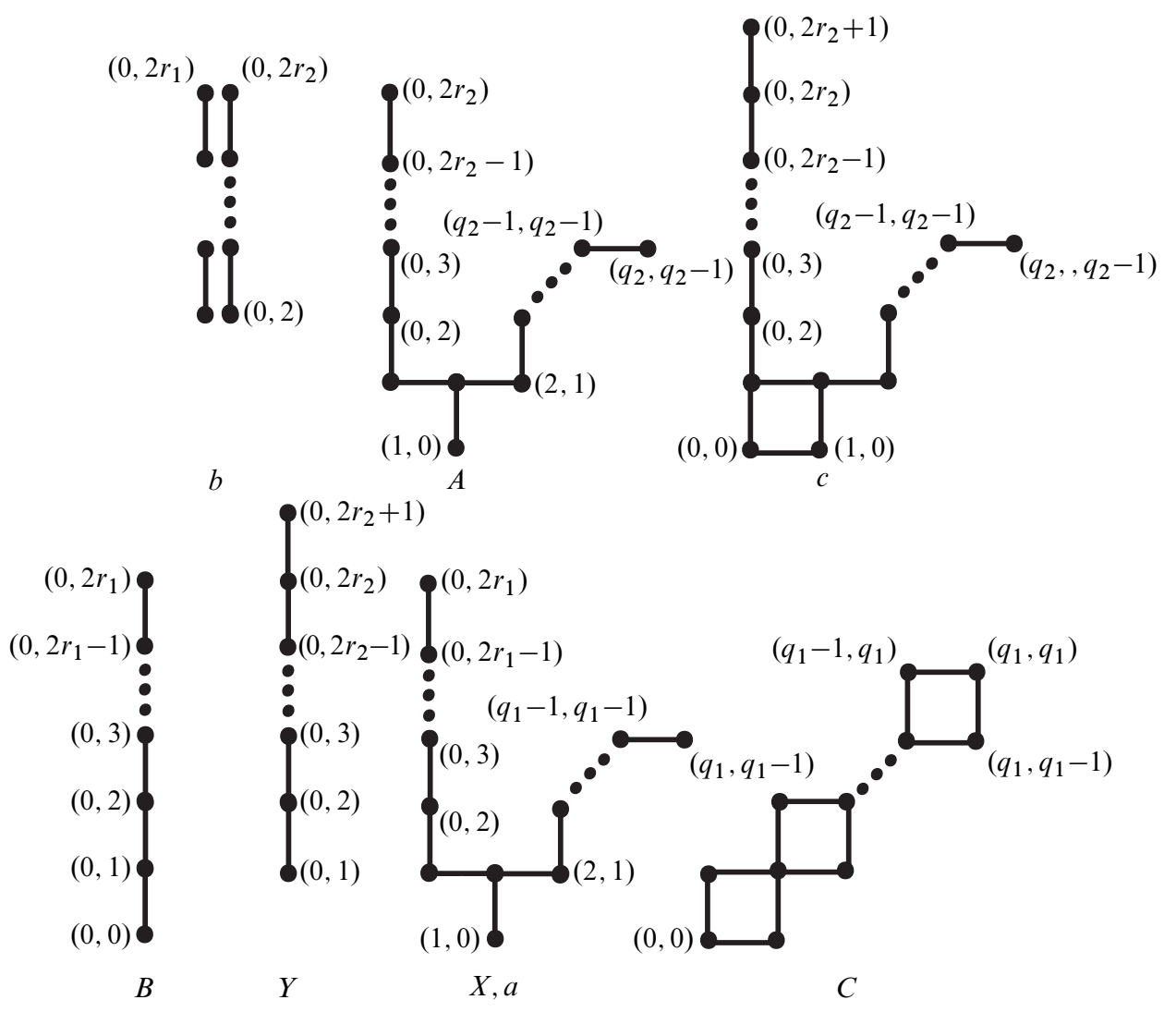

Figure 18: Filtration data for the eight types of intersection points in the type of Heegaard diagram shown in Figure 17

establishes the width of the polytope as $q_{1}+q_{2}$, and so we fix the horizontal coordinates for the new diagram as $0 \leq x \leq q_{1}+q_{1}$. On the other hand, the maximal $y$ value on the line $x=q_{1}+q_{2}$ has a single generator for both diagrams, so this lattice point in the new diagram is identified with $\left(q_{1}+q_{2}, q_{1}+q_{2}+2 r_{B}-1\right)$.

In the chain complex coming from the diagram formed by handleslides, the filtration level $\left(q_{1}+q_{2}, q_{1}+q_{2}-2\right)$ has two generators connected by a domain which is a square with four acute corners. This simply-connected domain is shown in Figure 20. Such a domain always supports a one-parameter family of holomorphic representatives, so the homology at this filtration level is trivial. (For a detailed discussion of square domains, see Sarkar and Wang [9].)

There are four generators in the filtration level $\left(q_{1}+q_{2}, q_{1}+q_{2}-1\right)$, as in the diagram introduced at the beginning of Section 6.2. Although the homology of this 

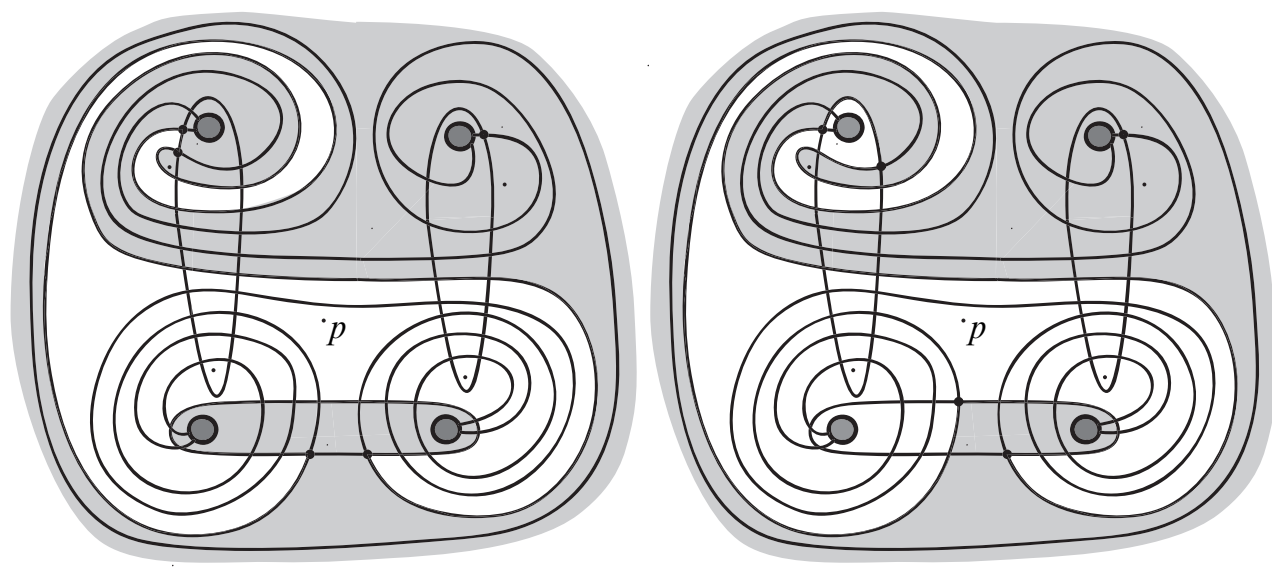

Figure 19: In filtration level $\left(q_{1}+q_{2}, q_{1}+q_{2}-1\right)$, the generator $\left(a_{7}, A_{7}, Y_{1}\right)$ cannot map to either $\left(A_{7}, B_{1}, C_{8}\right)$ (left) or $\left(A_{7}, B_{2}, C_{7}\right)$ (right), so the homology is has rank two.

subcomplex was difficult to compute in the original Heegaard diagram, on the new diagram one can show that $\widehat{\operatorname{HFL}}\left(P_{q_{1}, r, q_{2}, r},\left(q_{1}+q_{2}, q_{1}+q_{2}-1\right)\right)$ has rank two. The chain complex has two generators in two different gradings, and proving that one of the top-graded generators maps to neither of the bottom-graded generators establishes that the homology of the subcomplex cannot be trivial. The relevant domains are indicated in Figure 19. In each case, there is an additional marked point $p$ with $n_{p}(\phi)<n_{z_{1}}(\phi)$. Furthermore, $p$ has trivial intersection number with a generator for the space of periodic domains, so the argument from the proof of Lemma 2 shows that no boundary disc connects these two generators. (One may also show that the other top-graded generator has a nontrivial boundary, but we do not need this fact to conclude that the homology is nontrivial.) When $r_{1} \neq r_{2}$, this proves Lemma 3 .

When $r_{1}=r_{2}$, we claim $\widehat{\operatorname{HFL}}\left(P_{q_{1}, r, q_{2}, r},\left(q_{1}+q_{2}, q_{1}+q_{2}+2 r-1\right)\right)$ also has rank two; there is a single pair of generators in this filtration level, and Figure 20 shows that no domain connecting them satisfies both the positivity and null-intersection conditions. Because the domain is not embedded in $\Sigma$, we indicate it by labelling the multiplicity of each component of $\Sigma-\left\{\alpha_{i}\right\}-\left\{\beta_{i}\right\}$. Nontrivial homology in this filtration level proves that the vertical edge has length $2 r$ for all values of $r$ and completes the proof.

As noted above, for a type II link with $r_{1}=r_{2}$, it remains to show that the homology at $\left(q_{1}+q_{2}-2, q_{1}+q_{2}+2 r-1\right)$ has rank two. The type of diagram constructed in the proof of Lemma 3 has two generators in this filtration level, and the domain shown 

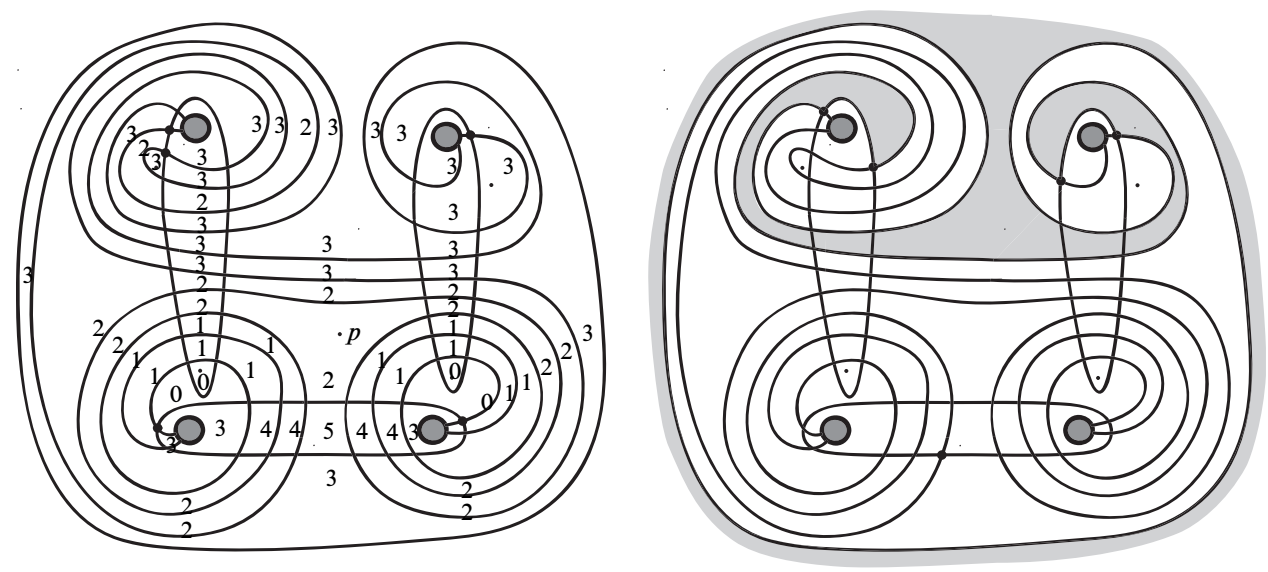

Figure 20: Left: The two generators in filtration level $\left(q_{1}+q_{2}, q_{1}+q_{2}+2 r-\right.$ 1) cannot be connected by a holomorphic disc. Right: The two generators in filtration level $\left(q_{1}+q_{2}, q_{1}+q_{2}-2\right)$ are joined by a simply-connected domain supporting a holomorphic disc, so the homology is trivial.

in Figure 21 proves that no disc connecting these generators can satisfy the positivity and null intersection conditions simultaneously. This completes the proof of Theorem 1 for all cases.

We note that for type I links with $r_{1} \neq r_{2}$, Lemma 3 may be deduced without resorting to the new diagram formed by handleslides. The minimal-complexity Seifert surface for $K$ constructed in Section 6.1 provides a lower bound for the Thurston norm of the class $(0,1)$ in $H_{2}\left(S^{3}, P_{q_{1}, r_{1}, q_{2}, r_{2}}\right)$. If the vertical edge of the Heegaard Floer polytope had length less than $2 r_{B}$, there would be a spanning surface for $K$ in $S^{3}-U$ with complexity less than that of the minimal-complexity Seifert surface for $K$ in $S^{3}$.

The Alexander polynomial of $P_{q_{1}, r_{1}, q_{2}, r_{2}}$ provides substantial, although not complete, information about the dual Thurston polytope when $r_{1} \neq r_{2}$. Because the points $\left(0,2 r_{1}+2 r_{2}+1\right)$ and $\left(q_{1}+q_{2}, q_{1}+q_{2}-1\right)$ have pairs of generators with relative grading one, they do not appear in the Newton polytope. However, the extreme points of $S$ where the rank of $\widehat{\mathrm{HFL}}\left(P_{q_{1}, r_{1}, q_{2}, r_{2}}, \mathbf{h}\right)$ is one correspond to terms in the Alexander polynomial with nonzero coefficients. In contrast, the Alexander polynomial reveals nothing about $B_{T}^{*}\left(P_{q, r}\right)$. Theorem 1 shows that despite this striking difference, minimal spanning surfaces in $P_{q, r}$ behave similarly to those in the general four-parameter family when $2 r_{S} \leq q_{S}-1$ : changing a single $r$-crossing changes the complexity of $F_{K}$ by two regardless of the twist numbers of the other columns. 


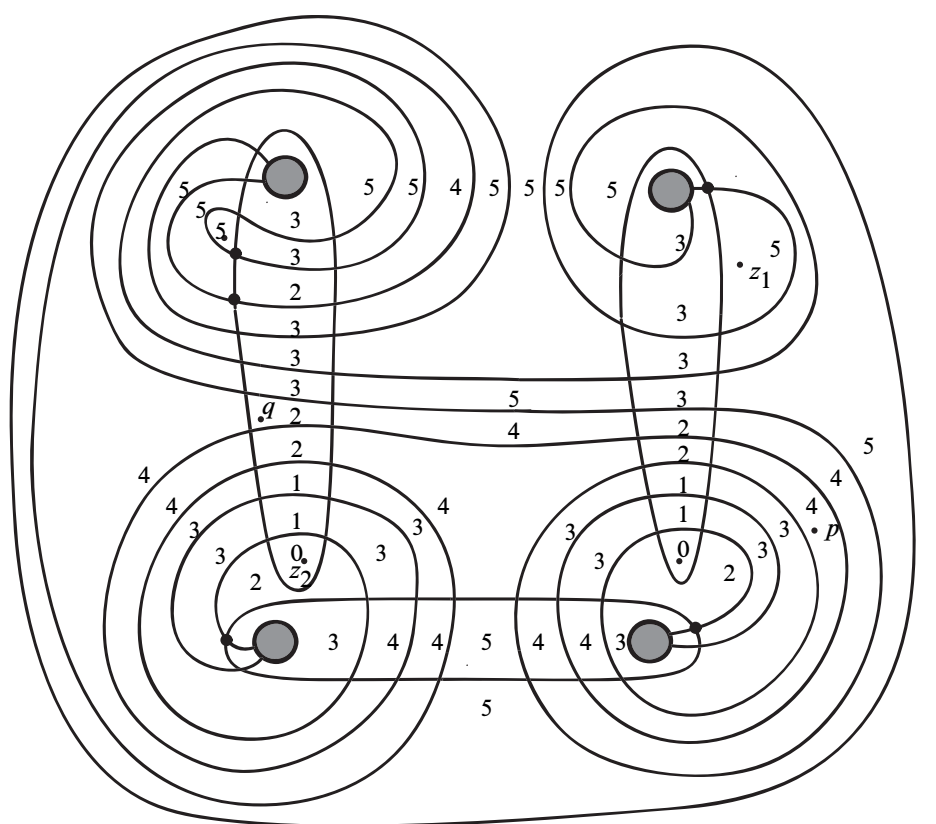

Figure 21: The integers on the diagram indicate the multiplicity of each component of $\Sigma-(\alpha \cup \beta)$ in a domain connecting the two generators in filtration level $\left(q_{1}+q_{2}-2, q_{1}+q_{2}+2 r-1\right) . n_{p}(\phi)<n_{z_{1}}(\phi)$, so there is no boundary disc from $\left(a_{6}, A_{6}, Y_{5}\right)$ to $\left(A_{6} . B_{5}, C_{6}\right)$.

\section{References}

[1] D Gabai, Foliations and the topology of 3-manifolds, J. Differential Geom. 18 (1983) 445-503

[2] R Lipshitz, A cylindrical reformulation of Heegaard Floer homology arXiv: math.SG/0502404

[3] C T McMullen, The Alexander polynomial of a 3-manifold and the Thurston norm on cohomology, Ann. Sci. École Norm. Sup. (4) 35 (2002) 153-171

[4] Y Ni, A note on knot Floer homology of links arXiv:math.GT/0506208

[5] PS Ozsváth, Z Szabó, Holomorphic disks, link invariants, and the multi-variable Alexander polynomial arXiv:math.GT/0512286

[6] PS Ozsváth, Z Szabó, Link Floer homology and the Thurston norm arXiv: math.GT/0601618

[7] P Ozsváth, Z Szabó, Holomorphic disks and knot invariants, Adv. Math. 186 (2004) $58-116$

Algebraic 83 Geometric Topology, Volume 8 (2008) 
[8] P Ozsváth, Z Szabó, Holomorphic disks and topological invariants for closed threemanifolds, Ann. of Math. (2) 159 (2004) 1027-1158

[9] S Sarkar, J Wang, A combinatorial description of some Heegaard Floer homologies arXiv:math.GT/0607777

[10] W P Thurston, A norm for the homology of 3-manifolds, Mem. Amer. Math. Soc. 59 (1986) i-vi and 99-130

Department of Mathematics, Stanford University

Stanford, CA 94305, USA

jelicata@stanford.edu

http://math.stanford.edu/ jelicata/

Received: 4 October $2006 \quad$ Revised: 16 August 2007 